\title{
A method for the stereoselective construction of the hemiaminal center in zampanolides
}

\author{
Tobias M. Brütsch, ${ }^{\#}$ Simone Berardozzi, ${ }^{\text {Marlene L. Rothe, }}$, \\ Mariano Redondo Horcajo, ${ }^{\$}$ José Fernando Díaz, ${ }^{\$}$ Karl-Heinz Altmann ${ }^{\#, *}$
}

"Department of Chemistry and Applied Biosciences, ETH-Zürich, Vladimir-Prelog-Weg 4, 8093 Zürich, Switzerland

${ }^{\$}$ Centro de Investigaciones Biológicas Margarita Salas, CSIC, Ramiro de Maetzu 9, 28040 Madrid, Spain

\section{Supporting Information \\ Spectra}




\section{${ }^{1} \mathrm{H}-\mathrm{NMR}$ compound 4}

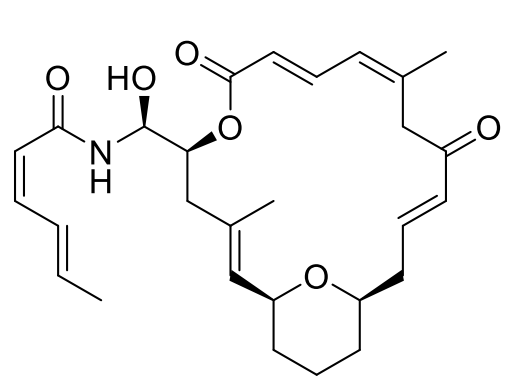

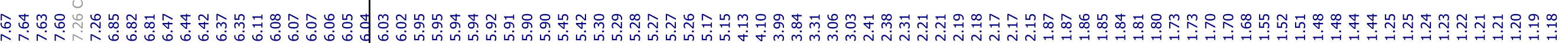
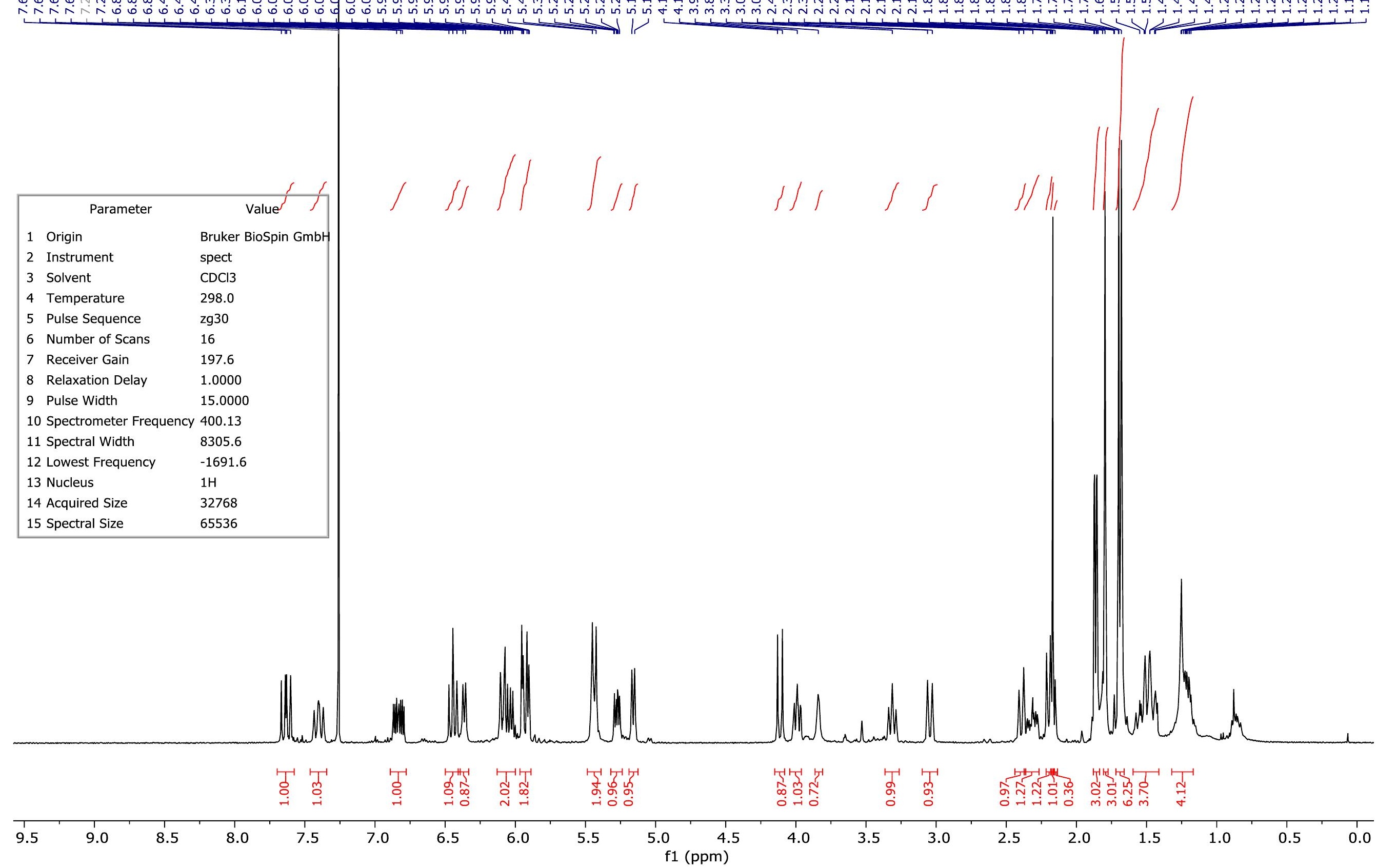
${ }^{13} \mathrm{C}-\mathrm{NMR}$ compound 4

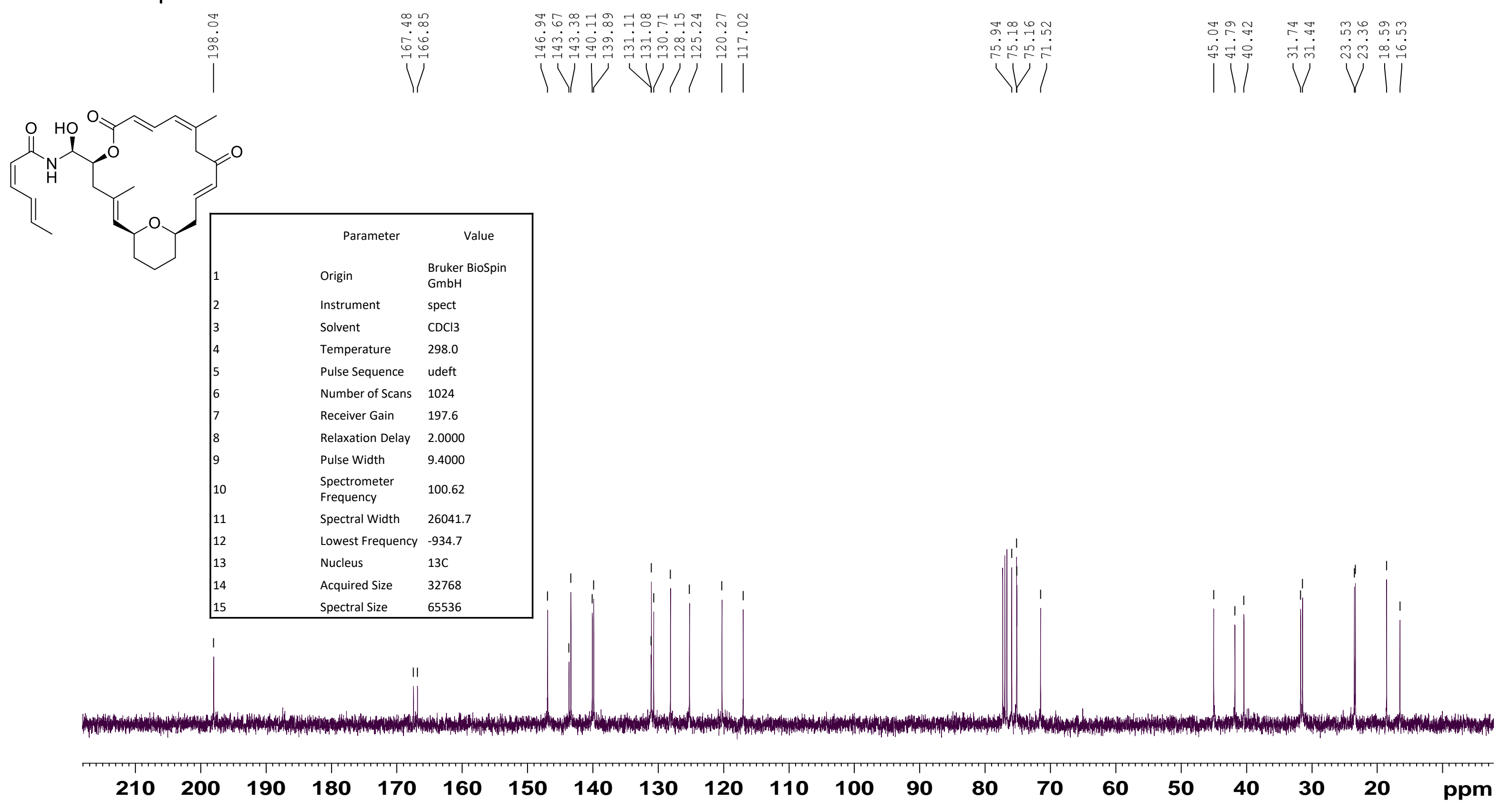




\section{${ }^{1} \mathrm{H}-\mathrm{NMR}$ compound epi-4}

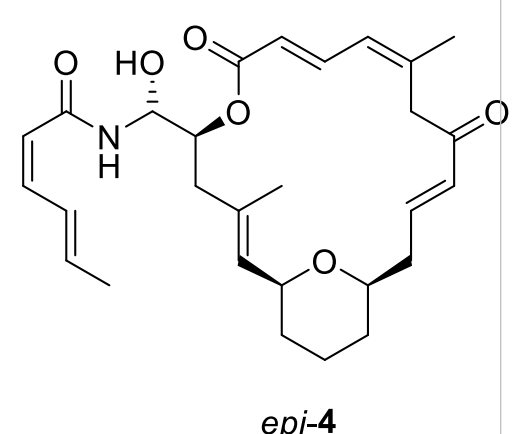

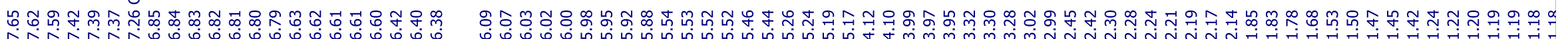

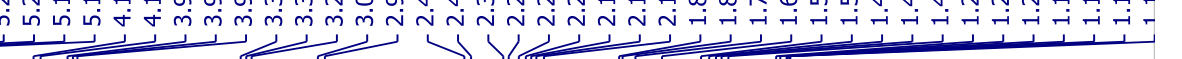

epi-4

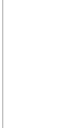

\begin{tabular}{|lll|}
\hline & & \multicolumn{2}{c|}{ Parameter } & \multicolumn{1}{c|}{ Value } \\
1 & Origin & Bruker BioSpin GmbH \\
2 & Instrument & spect \\
3 & Solvent & CDCl3 \\
4 & Temperature & 298.0 \\
5 & Pulse Sequence & $\mathrm{zg} 30$ \\
6 & Number of Scans & 256 \\
7 & Receiver Gain & 32.0 \\
8 & Relaxation Delay & 1.0000 \\
9 & Pulse Width & 4.5000 \\
10 & Spectrometer Frequency & 500.13 \\
11 & Spectral Width & 10330.6 \\
12 & Lowest Frequency & -2089.5 \\
13 & Nucleus & $1 \mathrm{H}$ \\
14 & Acquired Size & 32768 \\
15 & Spectral Size & 65536 \\
\hline
\end{tabular}

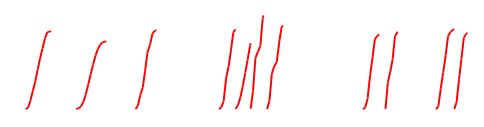

11

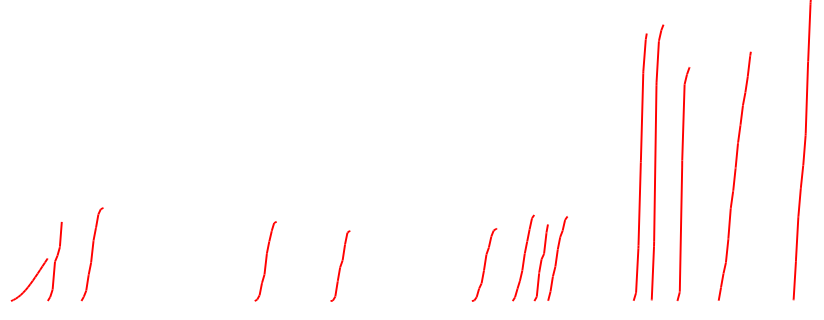

15 Spectral Size $\quad 65536$

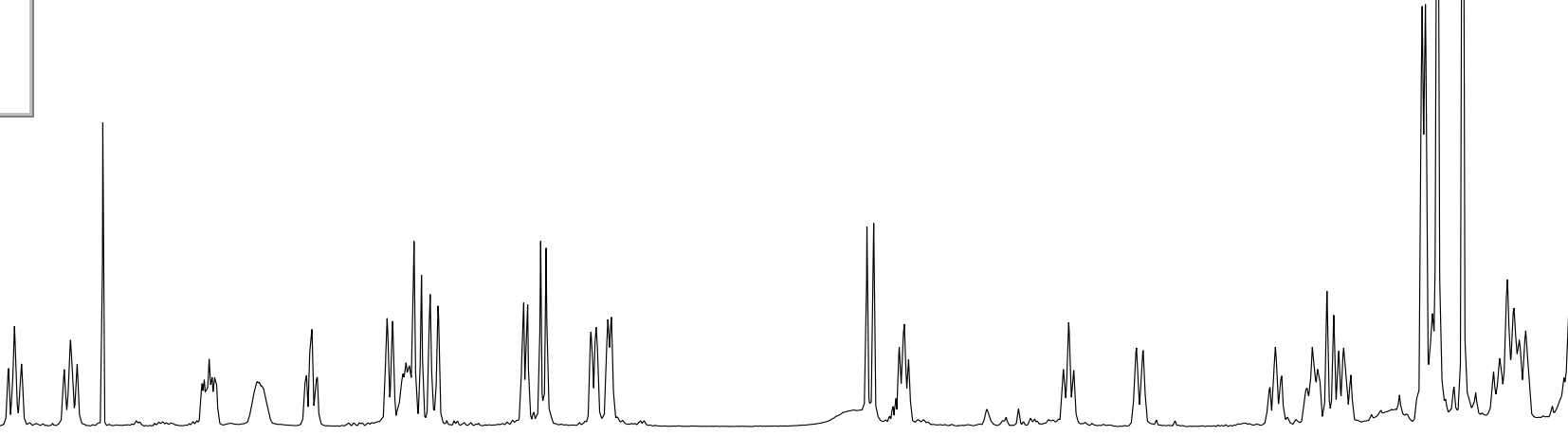

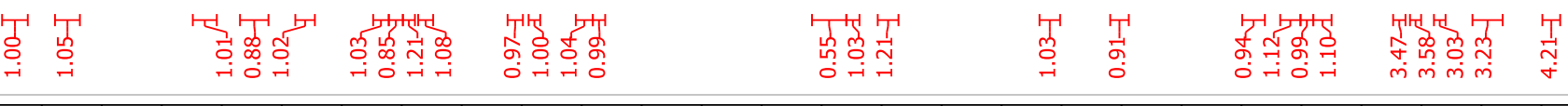

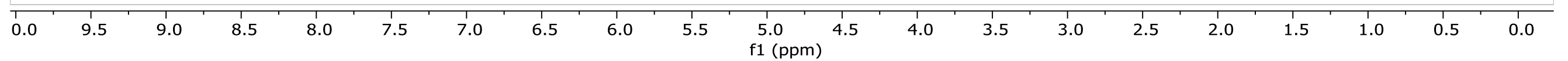




\section{${ }^{13} \mathrm{C}-\mathrm{NMR}$ compound epi-4}
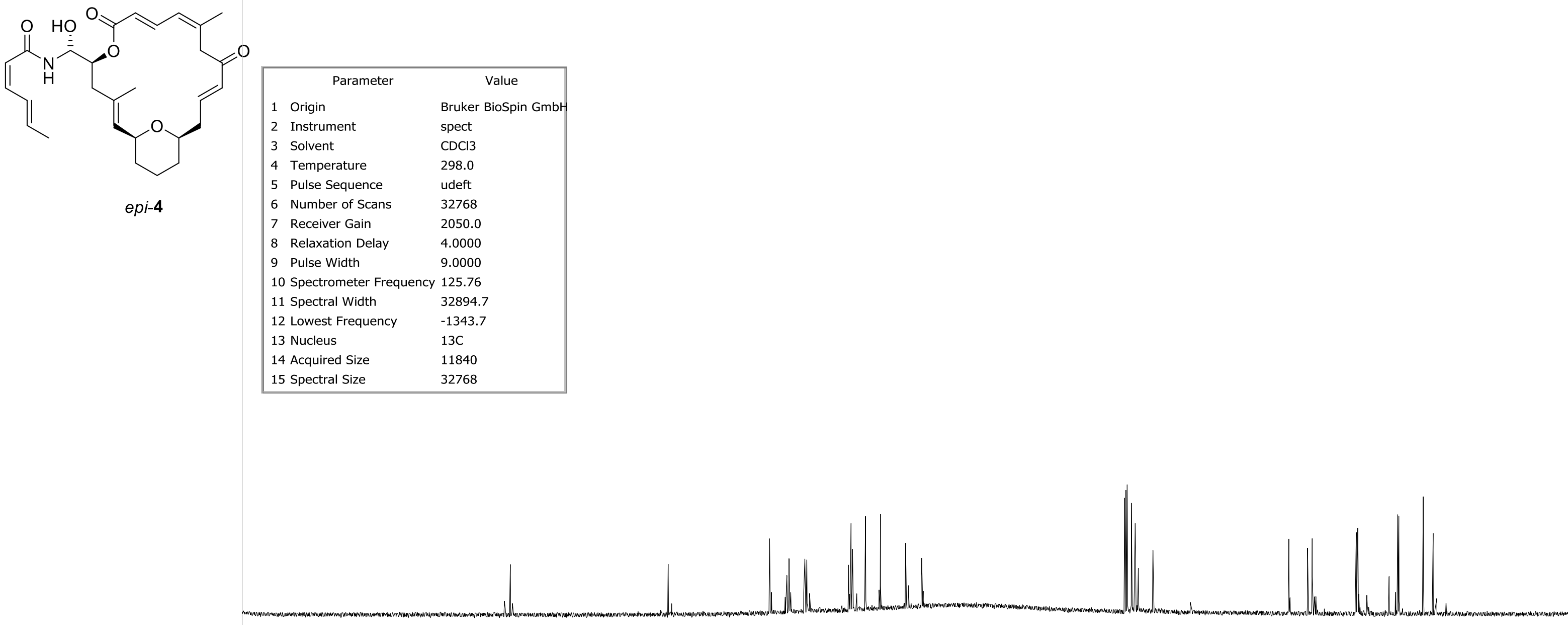


\section{${ }^{1} \mathrm{H}-\mathrm{NMR}$ compound $\mathbf{1}$}

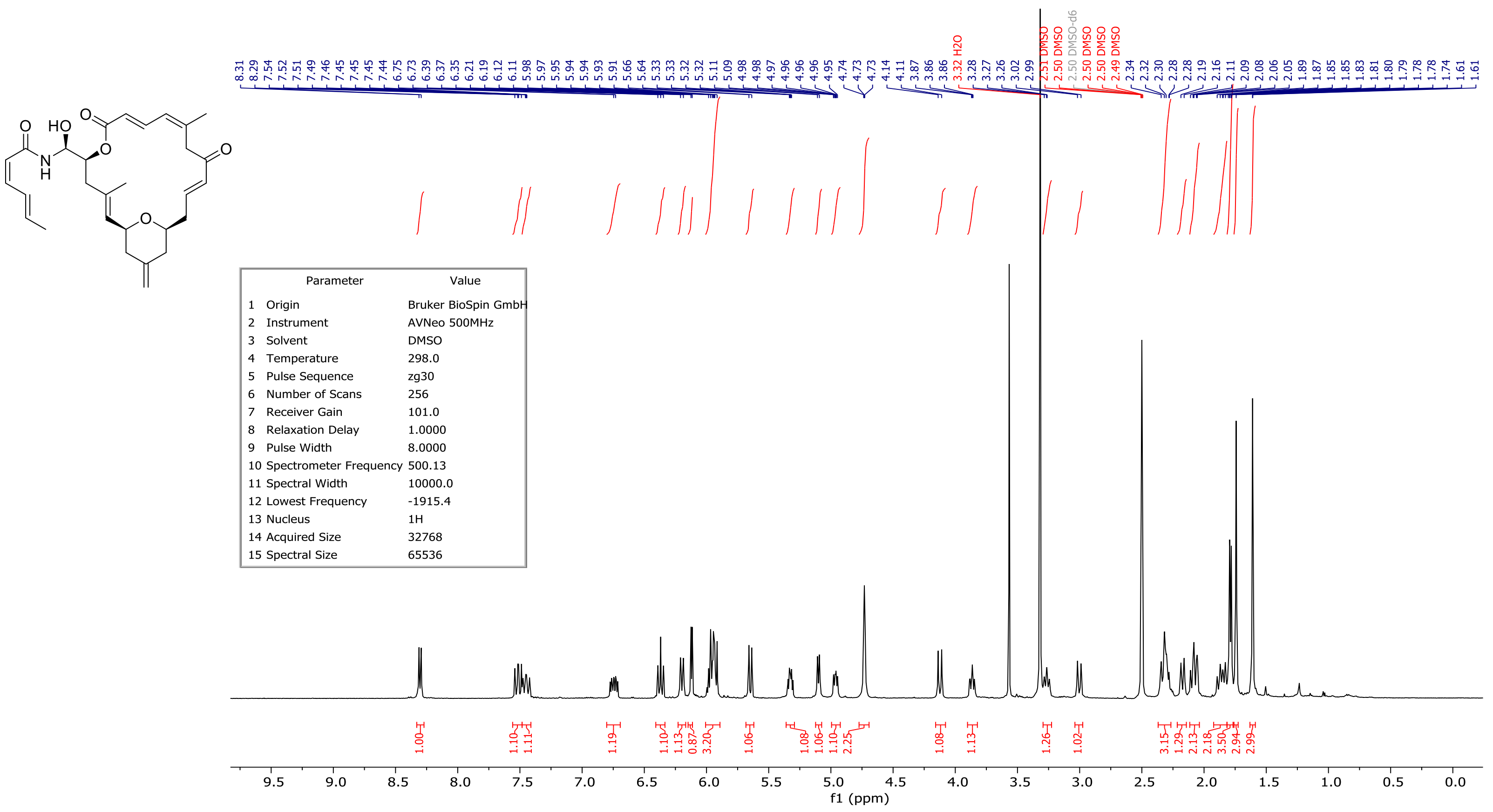




\section{${ }^{13} \mathrm{C}-\mathrm{NMR}$ compound 1}

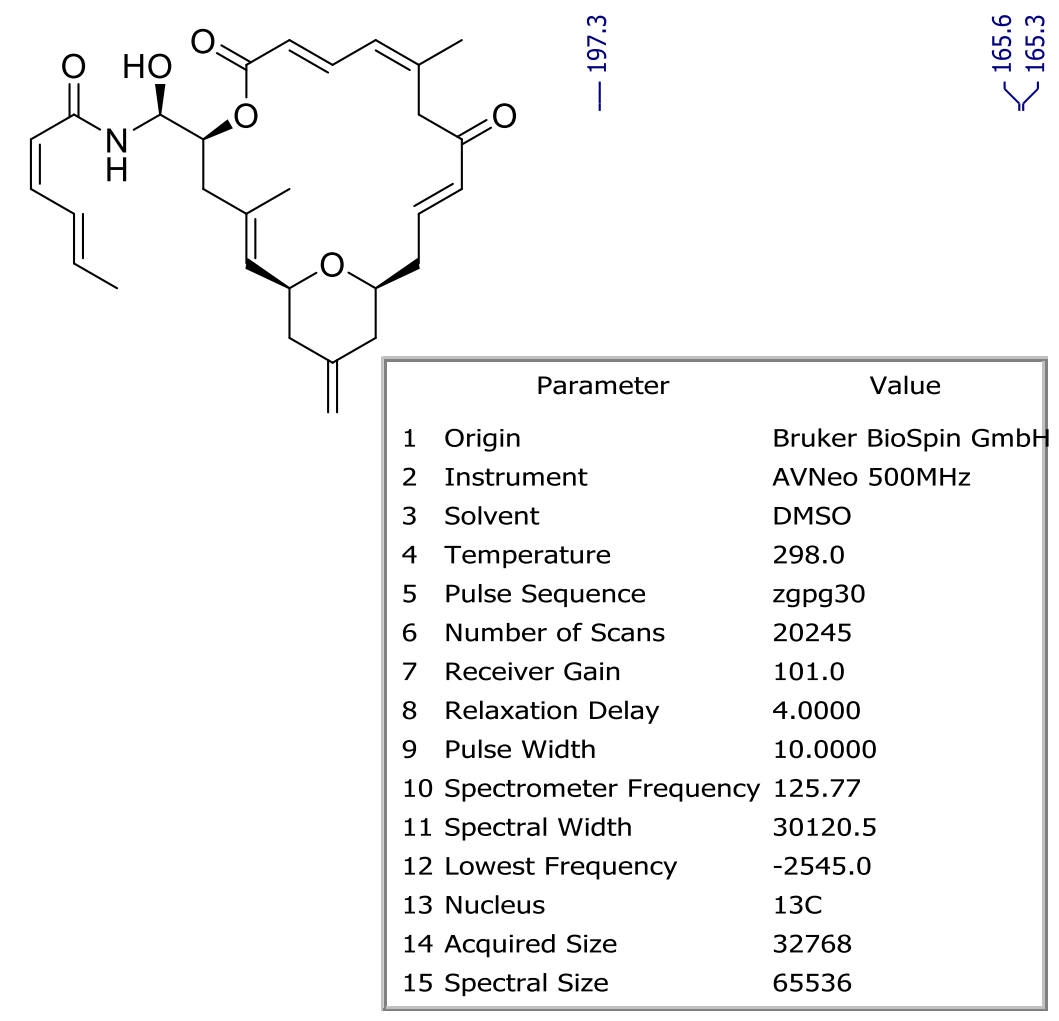

ctral Size 


\section{${ }^{1} \mathrm{H}-\mathrm{NMR}$ compound SI-2}

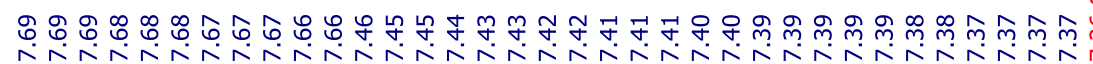
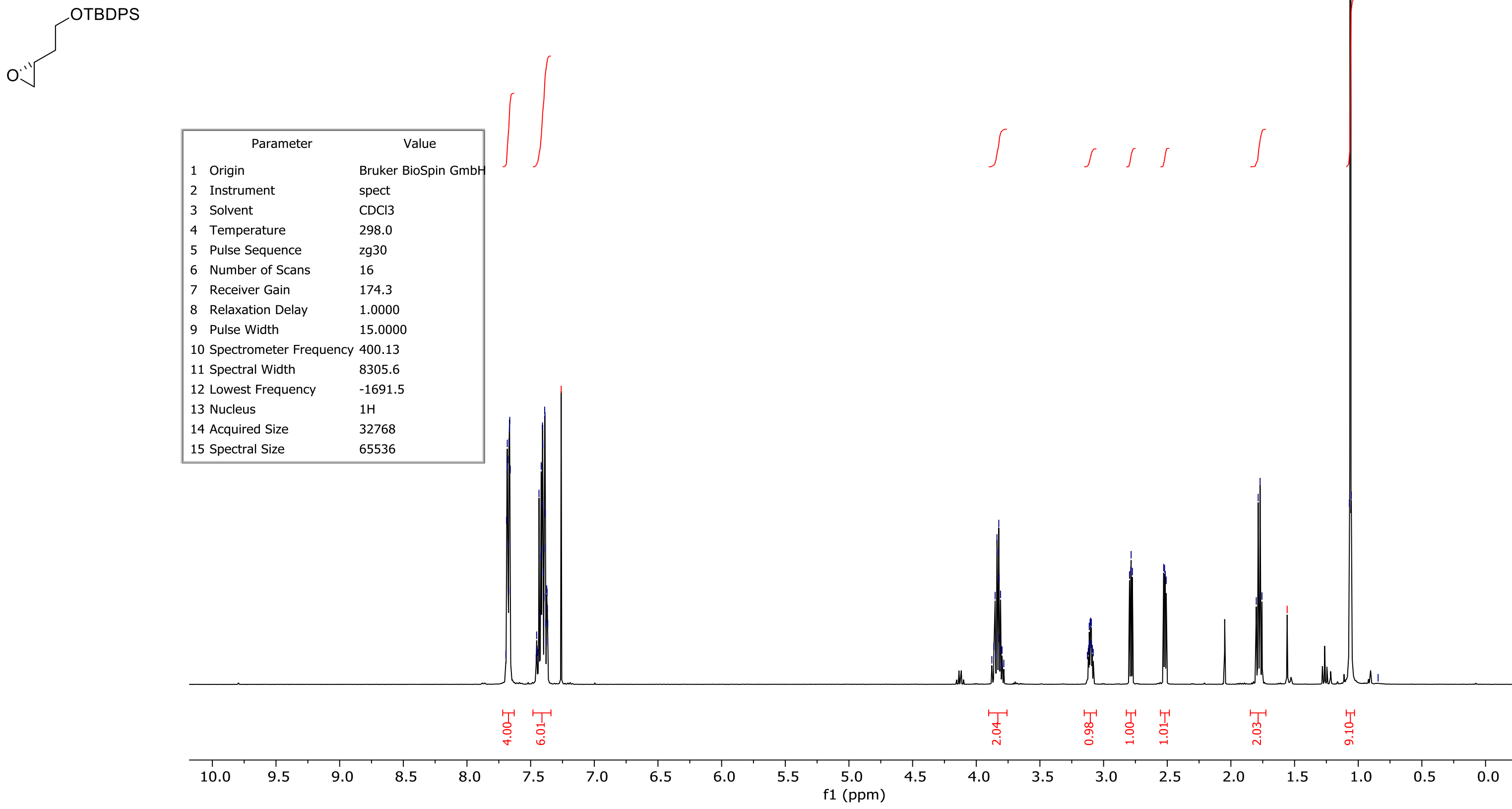


\section{${ }^{13} \mathrm{C}-\mathrm{NMR}$ compound SI-2}

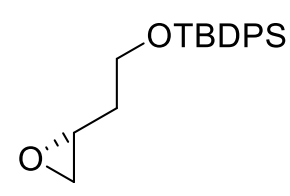

\begin{tabular}{|lll|}
\hline \multicolumn{1}{|c|}{ Parameter } & \multicolumn{1}{c|}{ Value } \\
1 & Origin & Bruker BioSpin \\
2 & Instrument & GmbH \\
3 & Solvent & CDCl \\
4 & Temperature & 298.0 \\
5 & Pulse Sequence & udeft \\
6 & Number of Scans & 256 \\
7 & Receiver Gain & 197.6 \\
8 & Relaxation Delay & 2.0000 \\
9 & Pulse Width & 9.4000 \\
10 & Spectrometer & 100.61 \\
$\quad$ Frequency & \\
11 & Spectral Width & 26041.7 \\
12 & Lowest Frequency & -934.1 \\
13 & Nucleus & $13 \mathrm{C}$ \\
14 & Acquired Size & 4096 \\
15 & Spectral Size & 32768 \\
\hline
\end{tabular}




\section{${ }^{1} \mathrm{H}-\mathrm{NMR}$ compound SI-3}

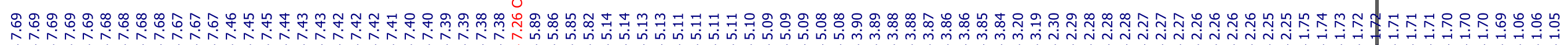

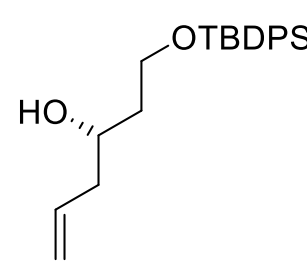

\begin{tabular}{|lll|}
\multicolumn{4}{|c|}{ Parameter } & \multicolumn{2}{c|}{ Value } \\
1 & Origin & Bruker BioSpin GmbH \\
2 & Instrument & spect \\
3 & Solvent & CDCl3 \\
4 & Temperature & 298.0 \\
5 & Pulse Sequence & zg30 \\
6 & Number of Scans & 16 \\
7 & Receiver Gain & 155.3 \\
8 & Relaxation Delay & 1.0000 \\
9 & Pulse Width & 15.0000 \\
10 & Spectrometer Frequency & 400.13 \\
11 & ppectral Width & 8305.6 \\
12 & Lowest Frequency & -1691.5 \\
13 & Nucleus & $1 \mathrm{H}$ \\
14 & Acquired Size & 32768 \\
15 & Spectral Size & 65536 \\
\hline
\end{tabular}$$
15 \text { Spectral Size }
$$

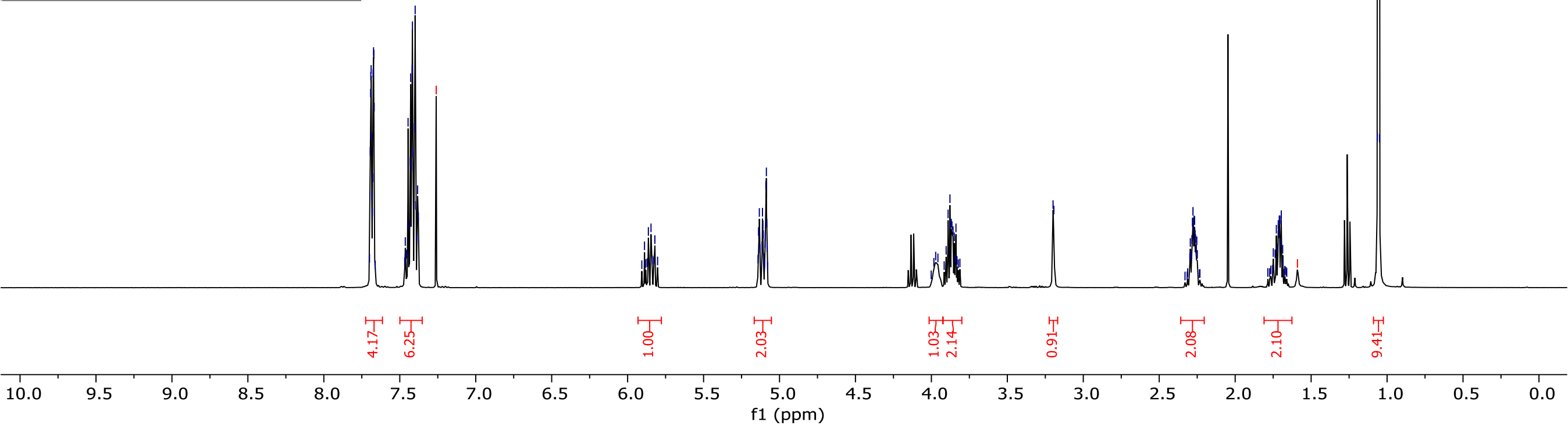




\section{${ }^{13} \mathrm{C}-\mathrm{NMR}$ compound $\mathrm{SI}-3$}

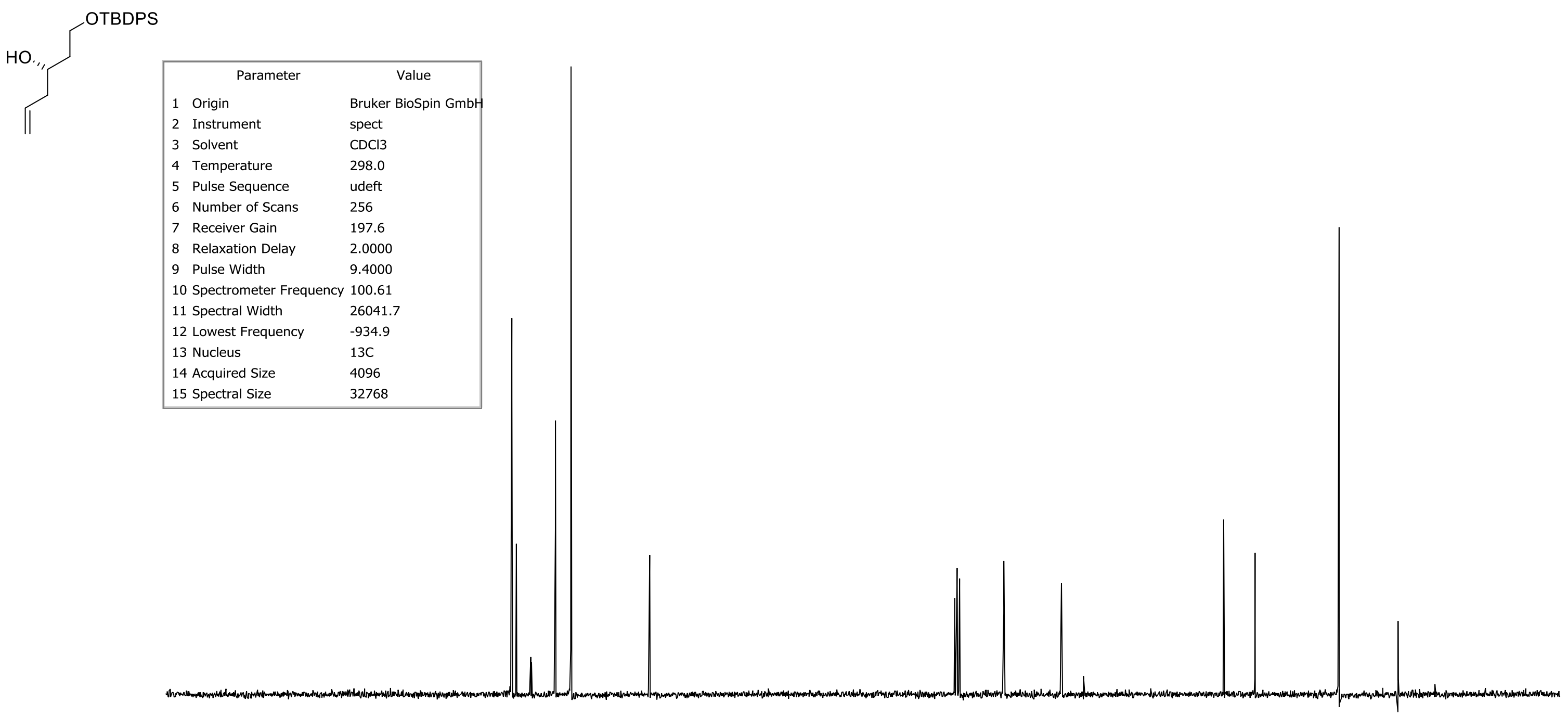




\section{${ }^{1} \mathrm{H}-\mathrm{NMR}$ compound SI-4}

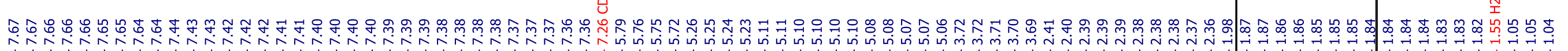
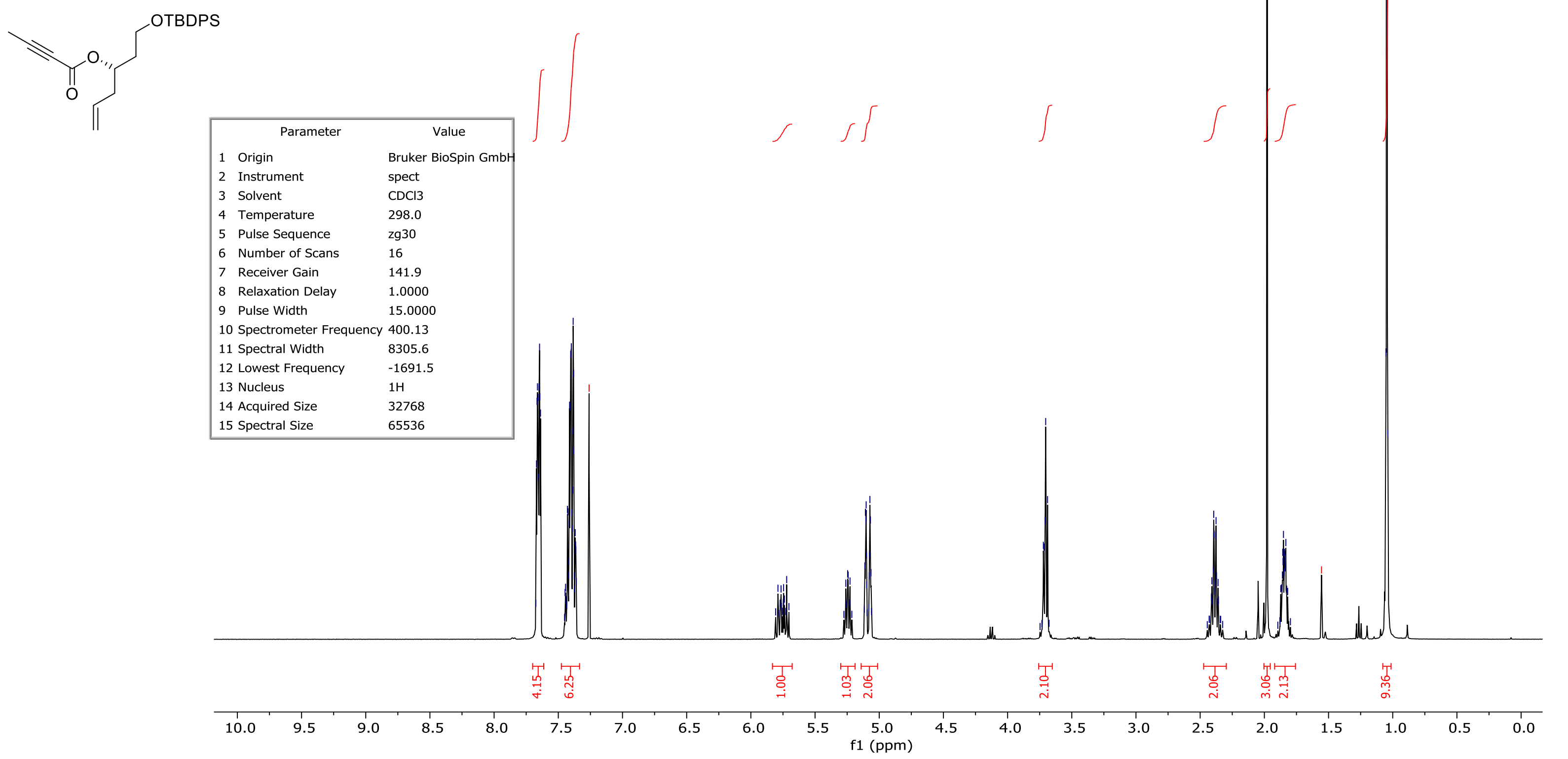


\section{${ }^{13} \mathrm{C}-\mathrm{NMR}$ compound $\mathrm{SI}-4$}

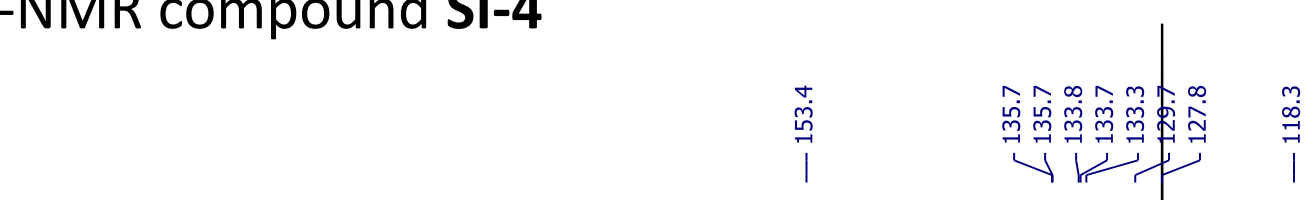

OTBDPS




\section{${ }^{1} \mathrm{H}-\mathrm{NMR}$ compound SI-5}

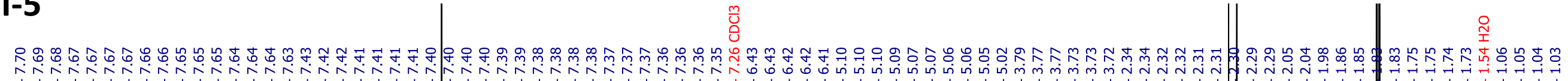
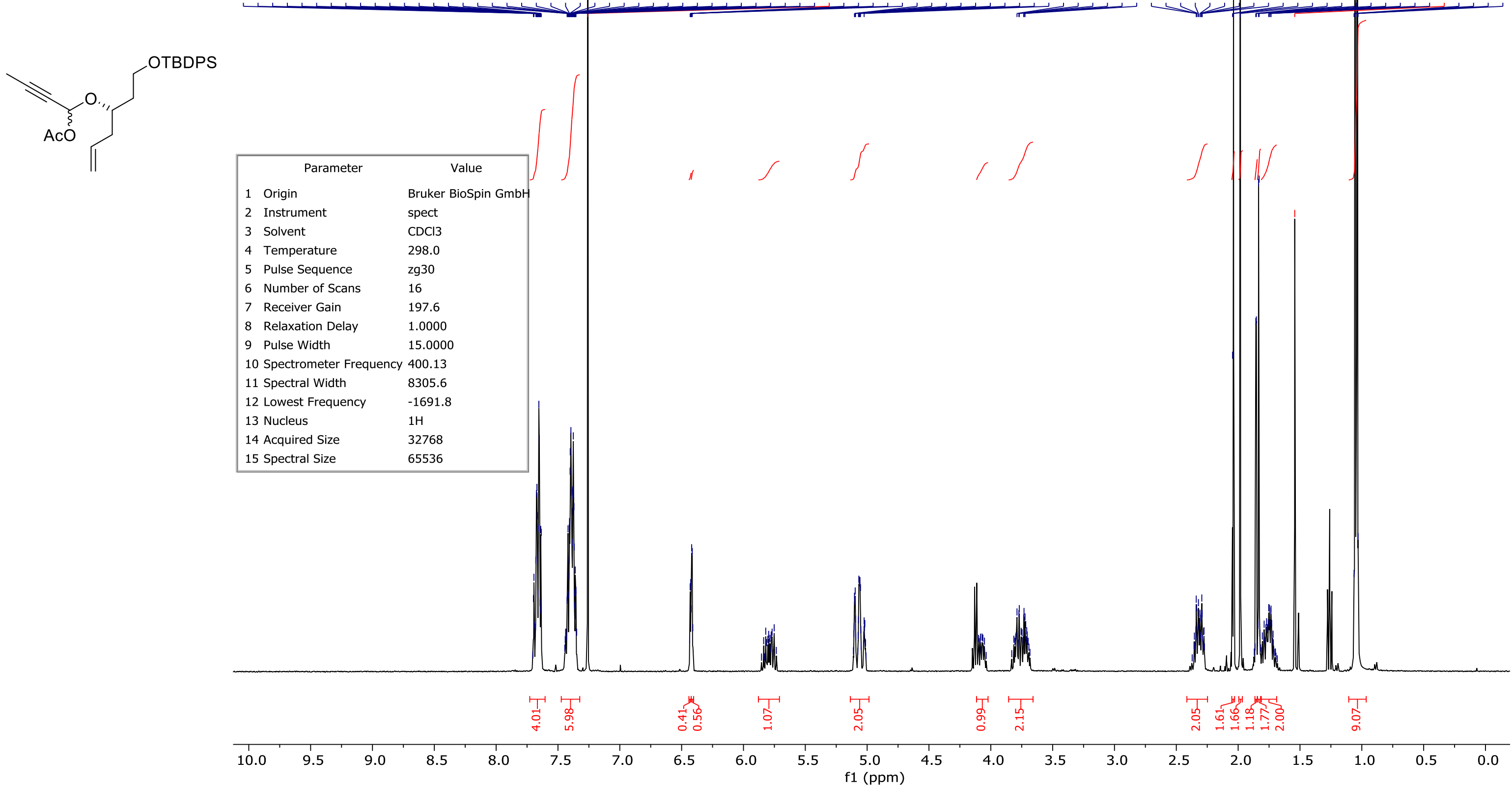
${ }^{13} \mathrm{C}-\mathrm{NMR}$ compound SI-5

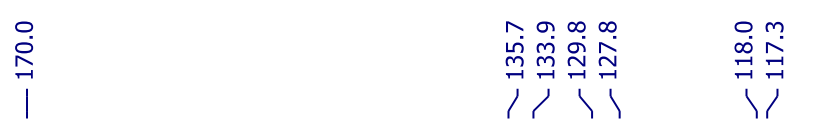

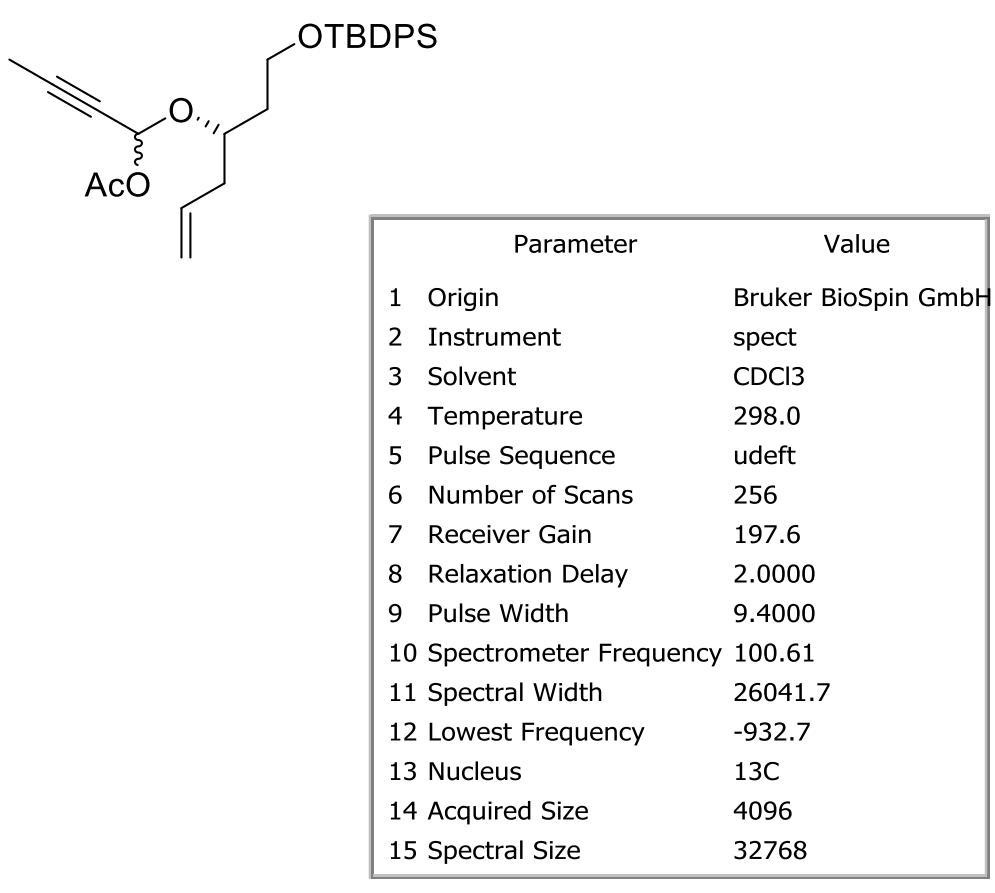

\begin{tabular}{|lll|}
\hline \multicolumn{1}{|c|}{ Parameter } & \multicolumn{2}{c|}{ Value } \\
1 & Origin & Bruker BioSpin GmbH \\
2 & Instrument & spect \\
3 & Solvent & CDCl3 \\
4 & Temperature & 298.0 \\
5 & Pulse Sequence & udeft \\
6 & Number of Scans & 256 \\
7 & Receiver Gain & 197.6 \\
8 & Relaxation Delay & 2.0000 \\
9 & Pulse Width & 9.4000 \\
10 & Spectrometer Frequency & 100.61 \\
11 & Spectral Width & 26041.7 \\
12 & Lowest Frequency & -932.7 \\
13 & Nucleus & $13 \mathrm{C}$ \\
14 & Acquired Size & 4096 \\
15 & Spectral Size & 32768 \\
\hline
\end{tabular}

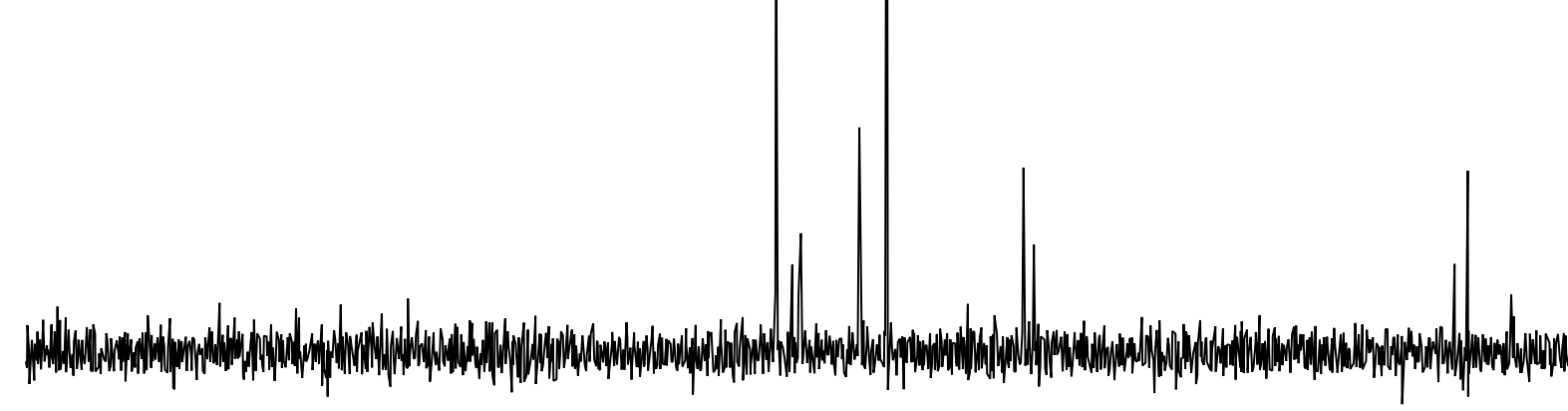




\section{${ }^{1} \mathrm{H}-\mathrm{NMR}$ compound SI-6}

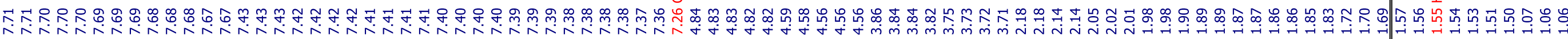

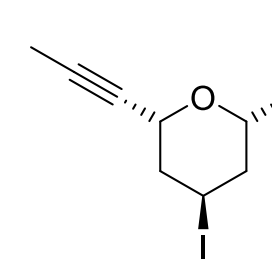
OTBDPS

\begin{tabular}{|lll|}
\hline \multicolumn{1}{|c|}{ Parameter } & \multicolumn{2}{c|}{ Value } \\
1 & Origin & Bruker BioSpin GmbH \\
2 & Instrument & spect \\
3 & Solvent & $\mathrm{CDCl}$ \\
4 & Temperature & 298.0 \\
5 & Pulse Sequence & $\mathrm{zg} 30$ \\
6 & Number of Scans & 16 \\
7 & Receiver Gain & 174.3 \\
8 & Relaxation Delay & 1.0000 \\
9 & Pulse Width & 15.0000 \\
10 & Spectrometer Frequency & 400.13 \\
11 & Spectral Width & 8305.6 \\
12 & Lowest Frequency & -1691.7 \\
13 & Nucleus & $1 \mathrm{H}$ \\
14 & Acquired Size & 32768 \\
15 & Spectral Size & 65536 \\
\hline
\end{tabular}

65536

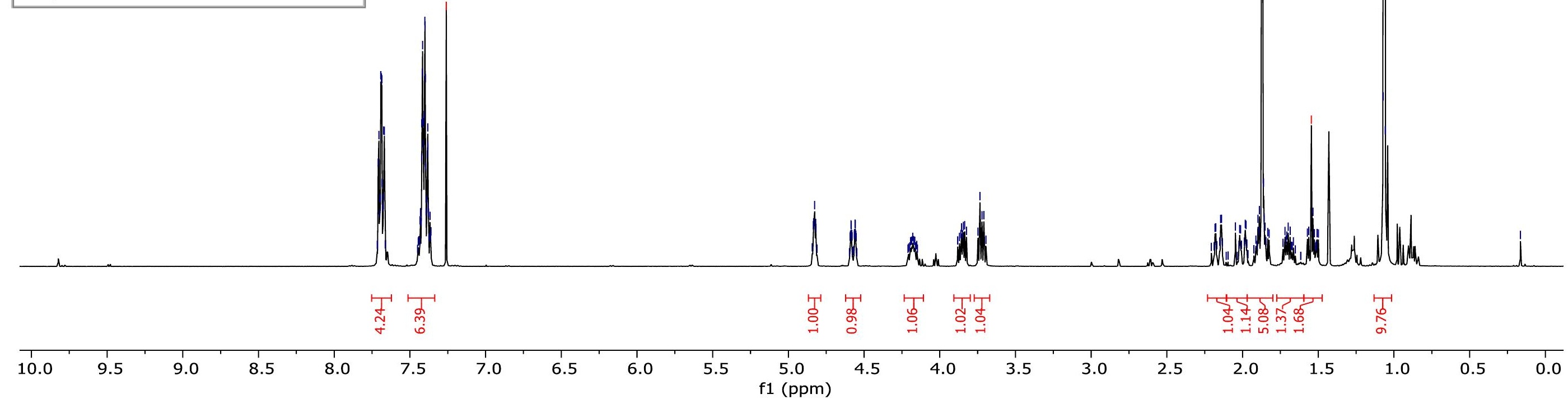




\section{${ }^{13}$ C-NMR compound SI-6}

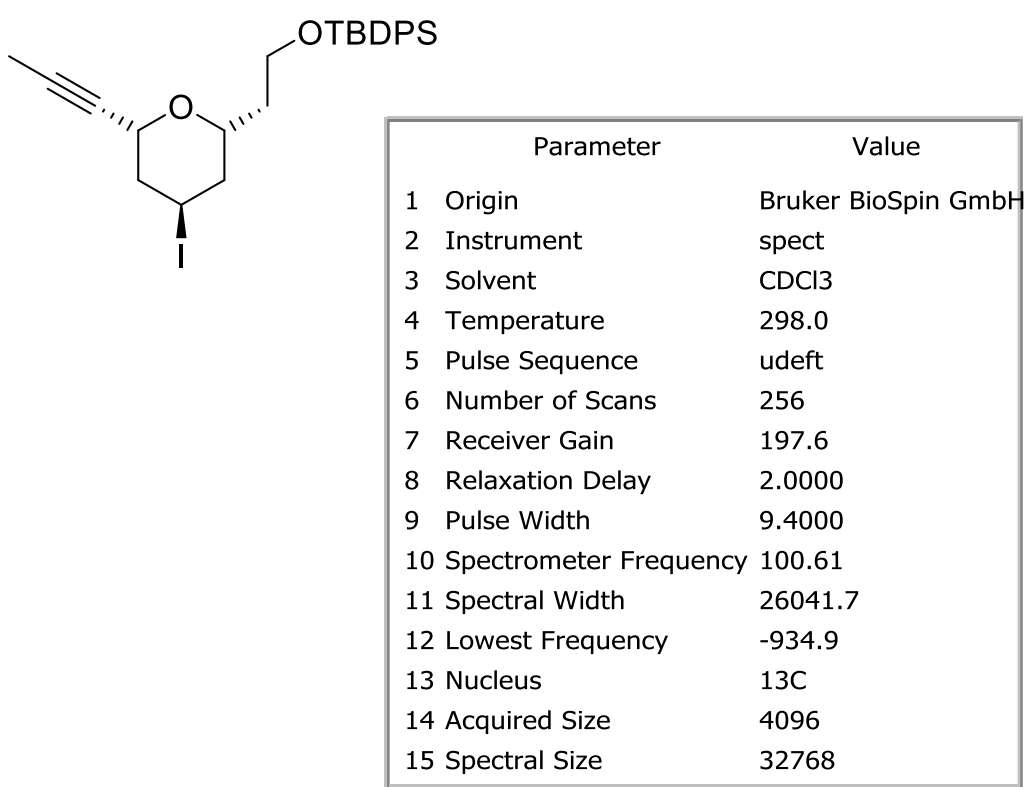

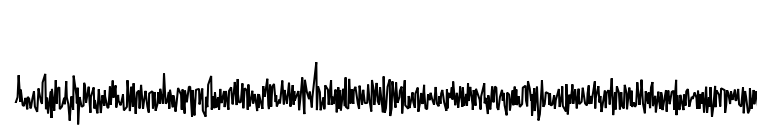

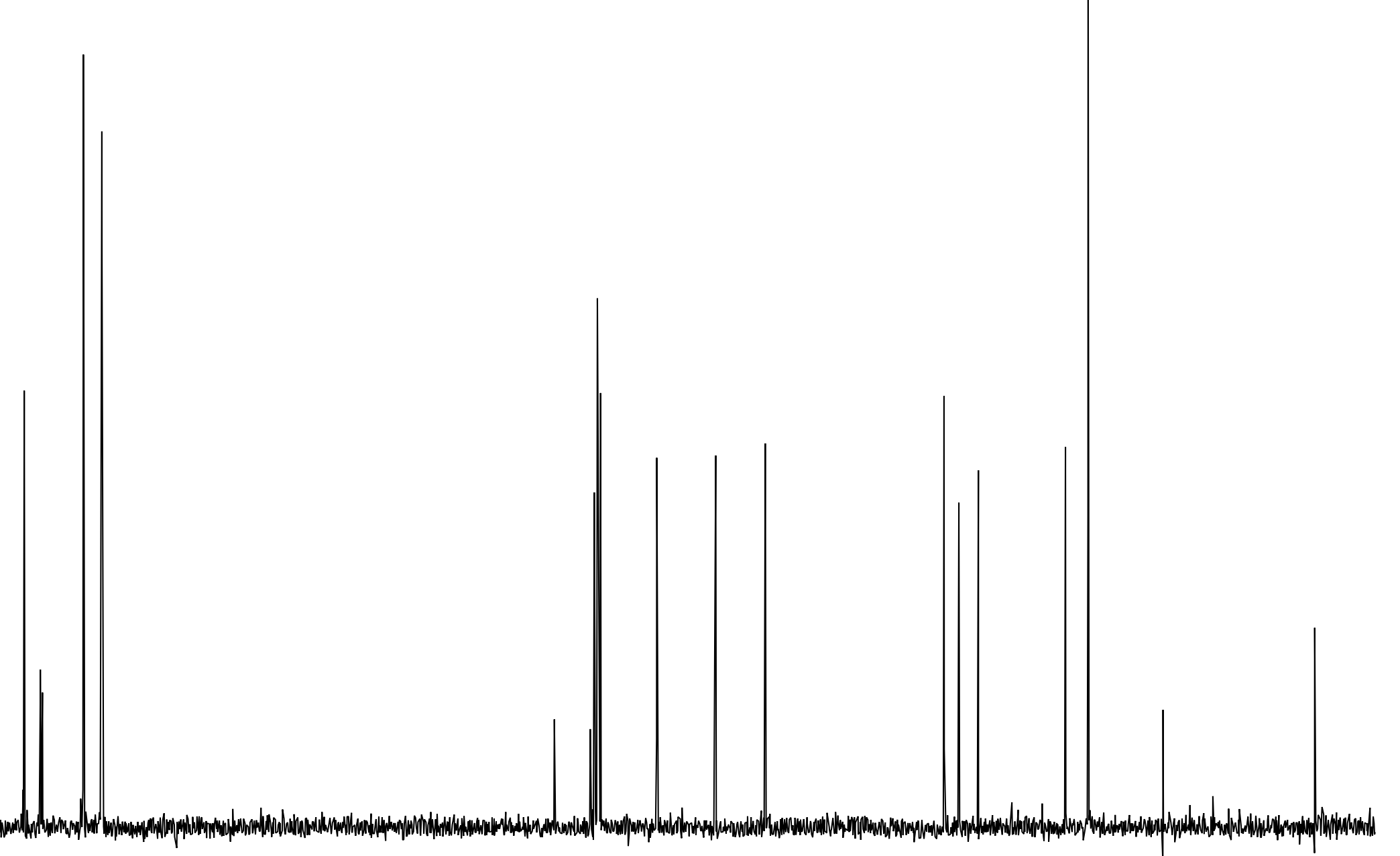




\section{${ }^{1} \mathrm{H}-\mathrm{NMR}$ compound SI-7}

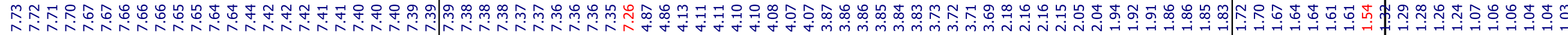
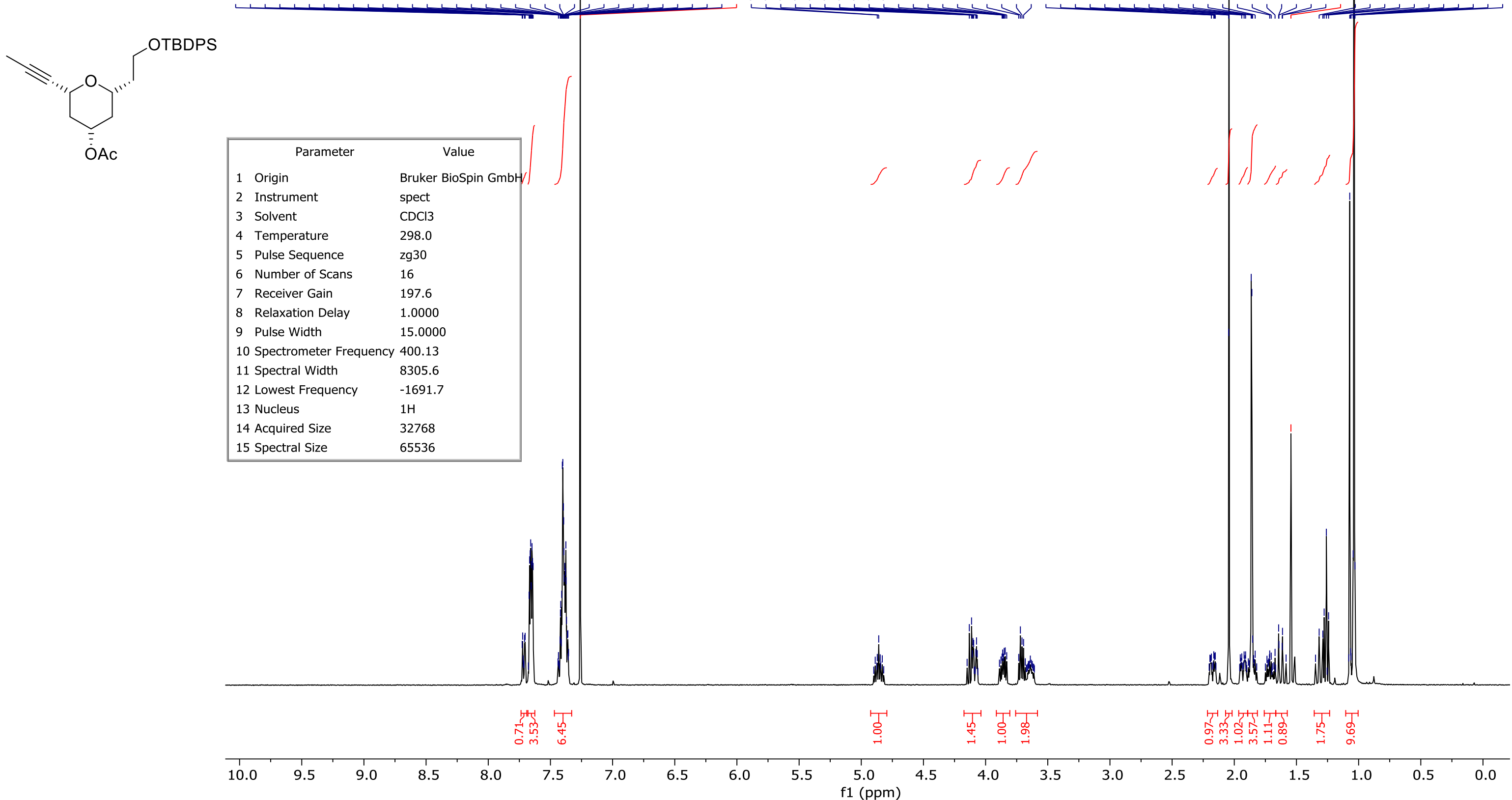


\section{${ }^{13}$ C-NMR compound SI-7}

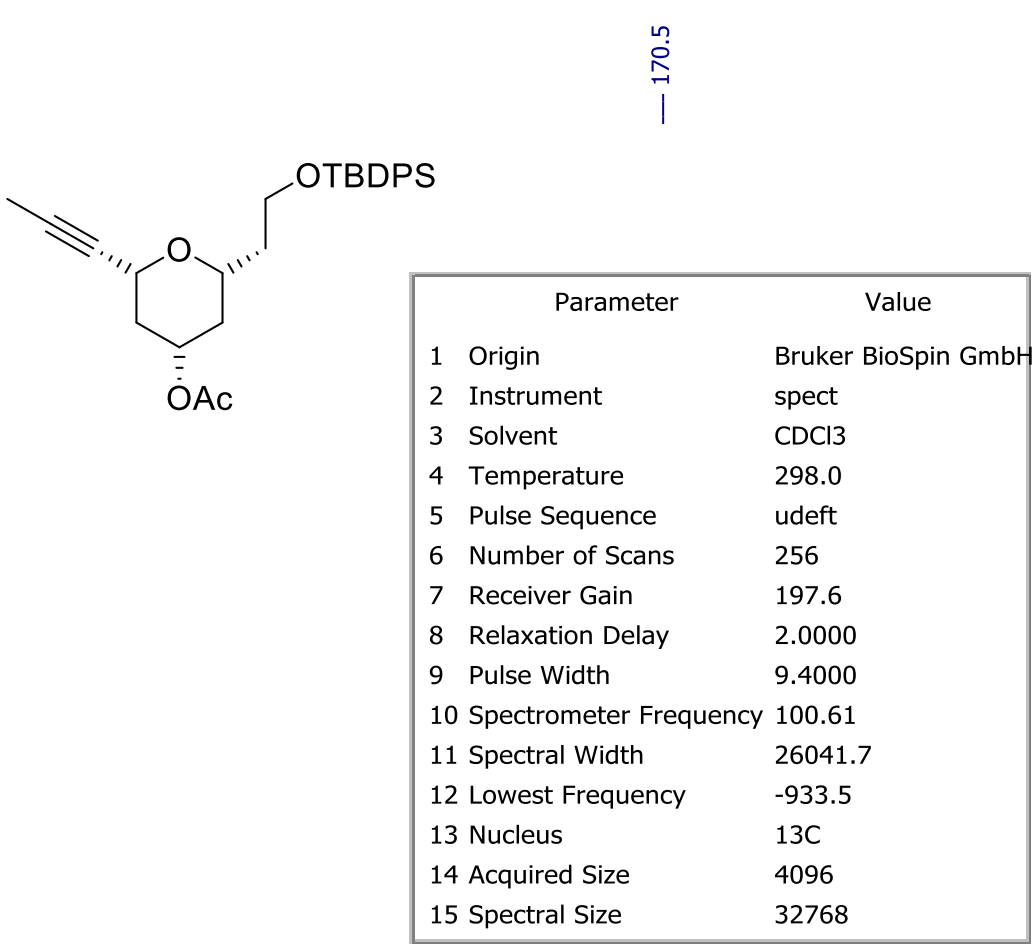




\section{${ }^{1} \mathrm{H}-\mathrm{NMR}$ compound SI-8}

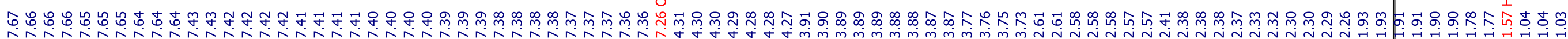

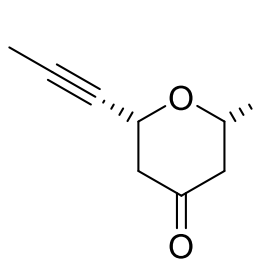

OTBDPS

\begin{tabular}{|lll|}
\hline \multicolumn{1}{|c|}{ Parameter } & \multicolumn{2}{c|}{ Value } \\
1 & Origin & Bruker BioSpin GmbH \\
2 & Instrument & spect \\
3 & Solvent & $\mathrm{CDCl} 3$ \\
4 & Temperature & 298.0 \\
5 & Pulse Sequence & $\mathrm{zg} 30$ \\
6 & Number of Scans & 16 \\
7 & Receiver Gain & 197.6 \\
8 & Relaxation Delay & 1.0000 \\
9 & Pulse Width & 15.0000 \\
10 & Spectrometer Frequency & 400.13 \\
11 & Spectral Width & 8305.6 \\
12 & Lowest Frequency & -1691.6 \\
13 & Nucleus & $1 \mathrm{H}$ \\
14 & Acquired Size & 32768 \\
15 & Spectral Size & 65536 \\
\hline
\end{tabular}

65536

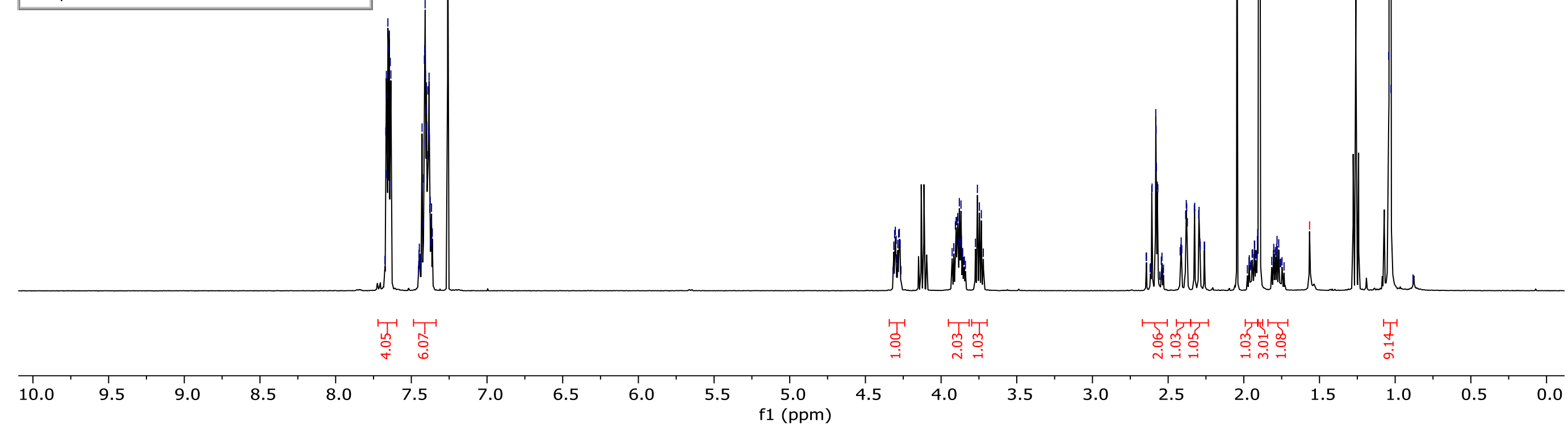




\section{${ }^{13} \mathrm{C}-\mathrm{NMR}$ compound SI-8}

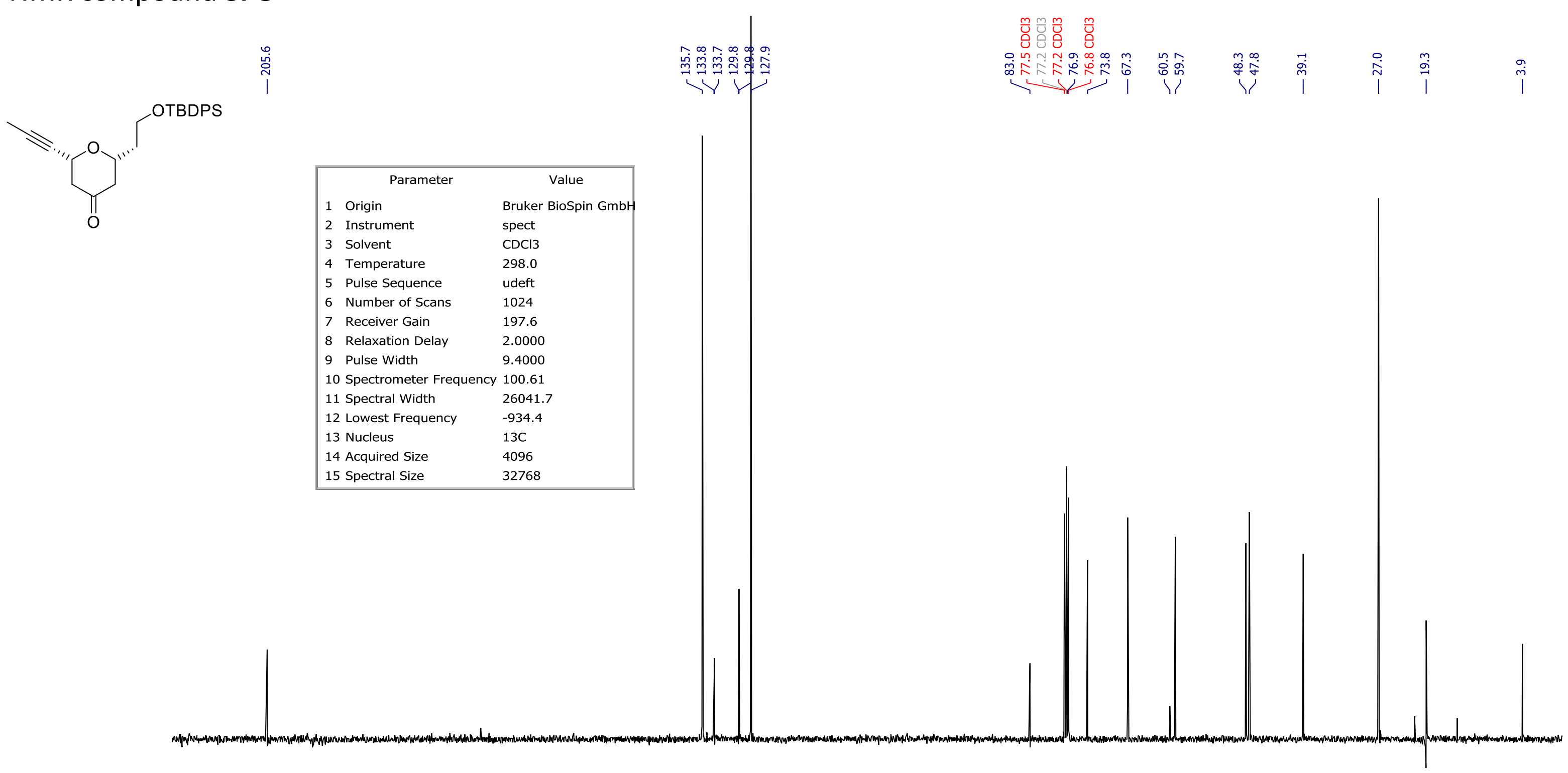




\section{${ }^{1} \mathrm{H}-\mathrm{NMR}$ compound SI-9}

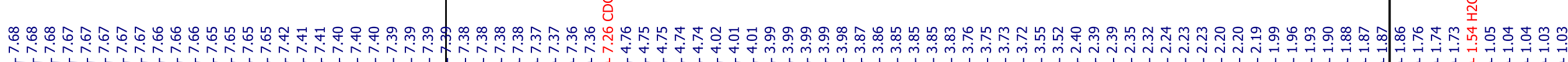

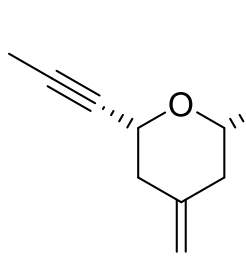

OTBDPS

\begin{tabular}{|lll|}
\hline \multicolumn{1}{|c|}{ Parameter } & \multicolumn{1}{c|}{ Value } \\
1 & Origin & Bruker BioSpin Gmb \\
2 & Instrument & spect \\
3 & Solvent & CDCl3 \\
4 & Temperature & 298.0 \\
5 & Pulse Sequence & zg30 \\
6 & Number of Scans & 16 \\
7 & Receiver Gain & 197.6 \\
8 & Relaxation Delay & 1.0000 \\
9 & Pulse Width & 15.0000 \\
10 & Spectrometer Frequency & 400.13 \\
11 & Spectral Width & 8305.6 \\
12 & Lowest Frequency & -1691.5 \\
13 & Nucleus & $1 \mathrm{H}$ \\
14 & Acquired Size & 32768 \\
15 & Spectral Size & 65536 \\
\hline
\end{tabular}
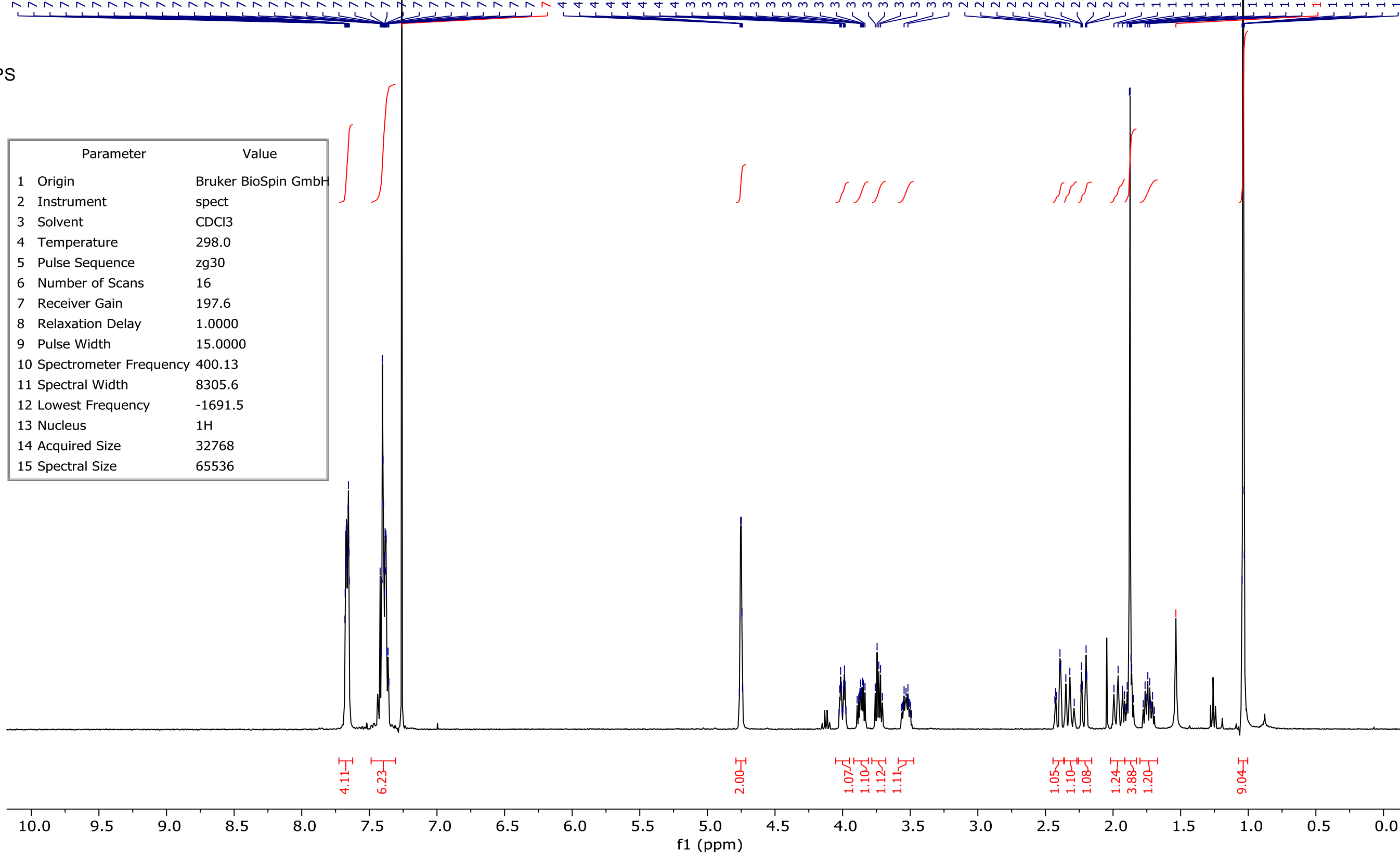

whe

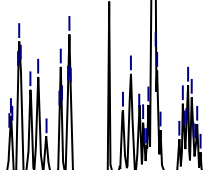




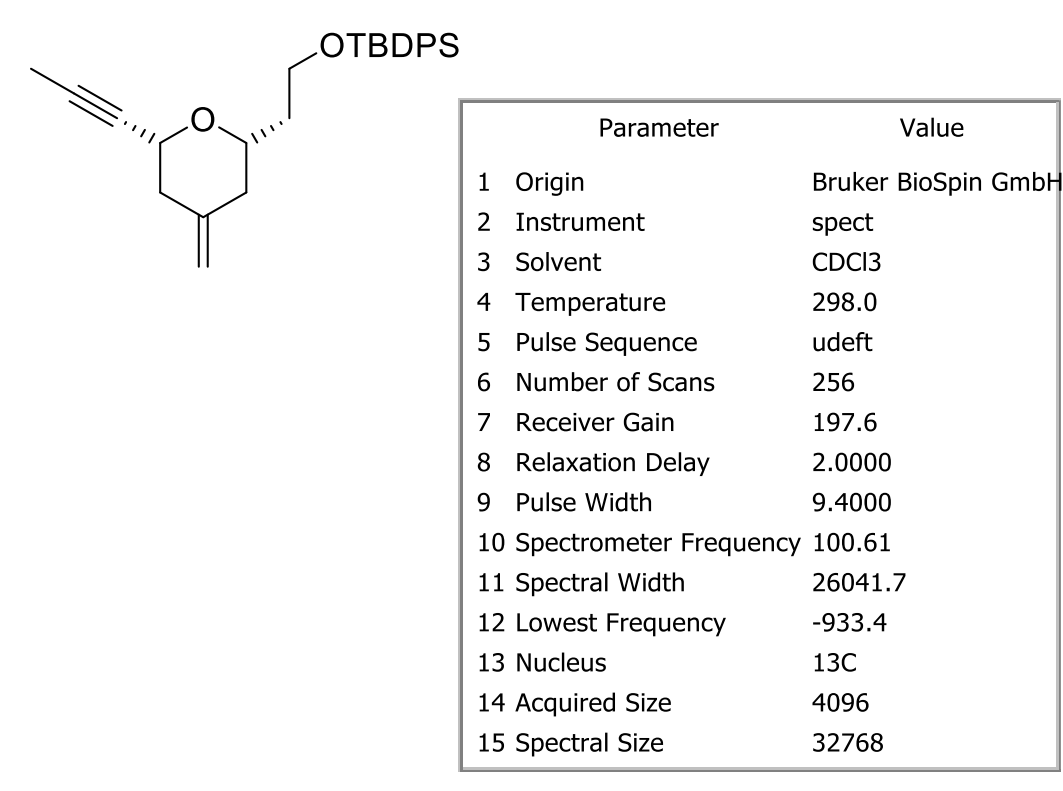

\begin{tabular}{|lll|}
\hline \multicolumn{1}{|c|}{ Parameter } & \multicolumn{1}{c|}{ Value } \\
1 & Origin & Bruker BioSpin GmbH \\
2 & Instrument & spect \\
3 & Solvent & CDCl3 \\
4 & Temperature & 298.0 \\
5 & Pulse Sequence & udeft \\
6 & Number of Scans & 256 \\
7 & Receiver Gain & 197.6 \\
8 & Relaxation Delay & 2.0000 \\
9 & Pulse Width & 9.4000 \\
10 & Spectrometer Frequency & 100.61 \\
11 & Spectral Width & 26041.7 \\
12 Lowest Frequency & -933.4 \\
13 & Nucleus & $13 C$ \\
14 & Acquired Size & 4096 \\
15 & Spectral Size & 32768 \\
\hline
\end{tabular}

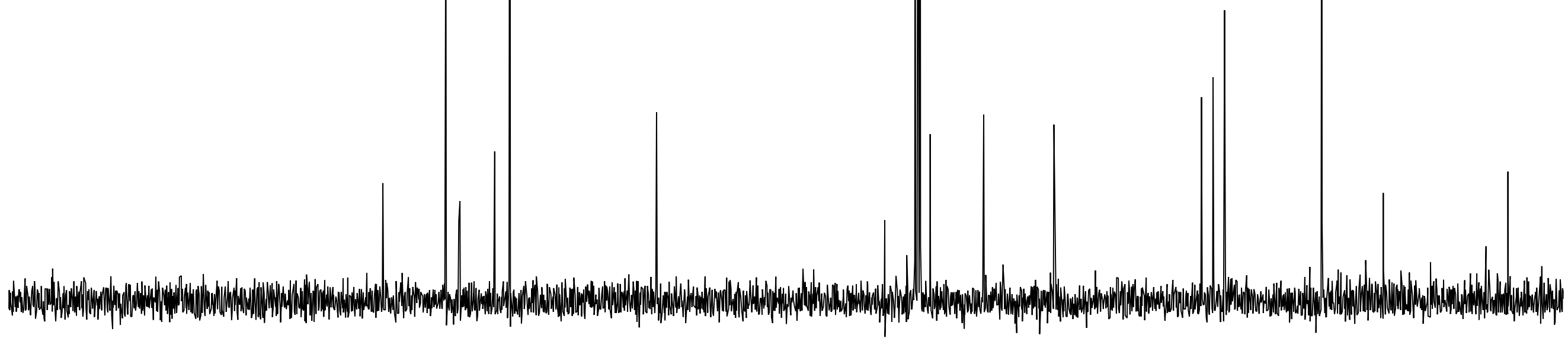




\section{${ }^{1} \mathrm{H}-\mathrm{NMR}$ compound $\mathrm{SI}-\mathbf{1 0}$}

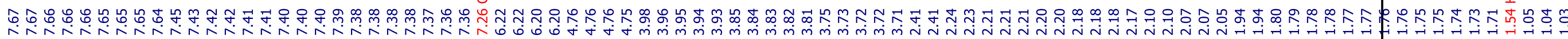

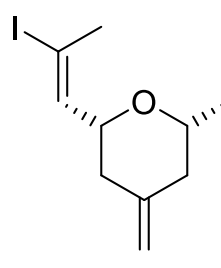

OTBDPS
\begin{tabular}{|lll|}
\hline \multicolumn{1}{|c|}{ Parameter } & \multicolumn{1}{c|}{ Value } \\
1 & Origin & Bruker BioSpin GmbH \\
2 & Instrument & spect \\
3 & Solvent & CDCl3 \\
4 & Temperature & 298.0 \\
5 & Pulse Sequence & zg30 \\
6 & Number of Scans & 16 \\
7 & Receiver Gain & 197.6 \\
8 & Relaxation Delay & 1.0000 \\
9 & Pulse Width & 15.0000 \\
10 & Spectrometer Frequency & 400.13 \\
11 & Spectral Width & 8305.6 \\
12 & Lowest Frequency & -1691.6 \\
13 & Nucleus & $1 \mathrm{H}$ \\
14 & Acquired Size & 32768 \\
15 & Spectral Size & 65536 \\
\hline
\end{tabular}

15 Spectral Size 65536

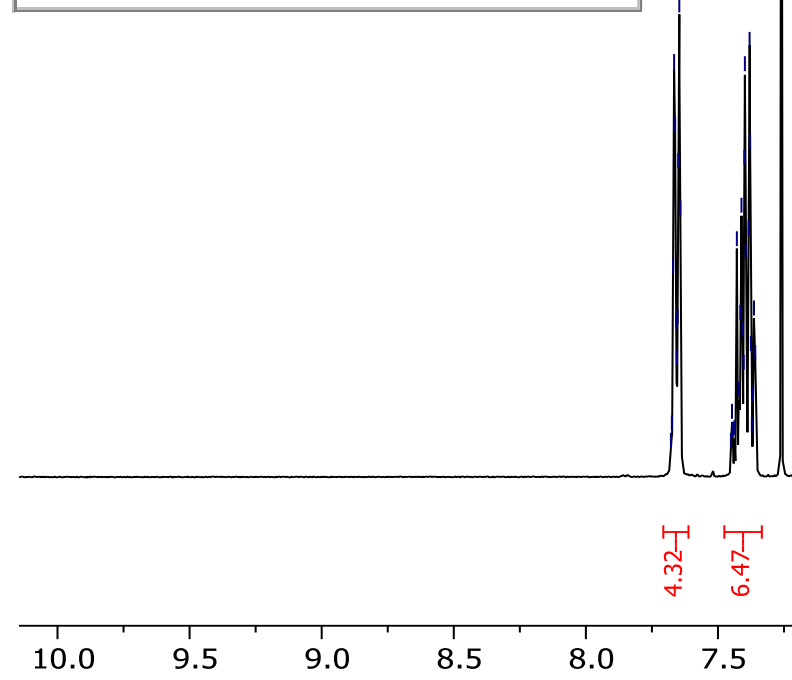

栏

Whollh

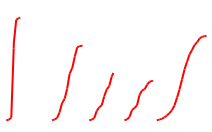




\begin{tabular}{|lll|}
\hline & \multicolumn{1}{c}{ Parameter } & \multicolumn{1}{c|}{ Value } \\
1 & Origin & Bruker BioSpin GmbH \\
2 & Instrument & spect \\
3 & Solvent & CDCI3 \\
4 & Temperature & 298.0 \\
5 & Pulse Sequence & udeft \\
6 & Number of Scans & 256 \\
7 & Receiver Gain & 197.6 \\
8 & Relaxation Delay & 2.0000 \\
9 & Pulse Width & 9.4000 \\
10 & Spectrometer Frequency & 100.61 \\
11 & Spectral Width & 26041.7 \\
12 & Lowest Frequency & -933.4 \\
13 & Nucleus & $13 \mathrm{C}$ \\
14 & Acquired Size & 4096 \\
15 & Spectral Size & 32768 \\
\hline
\end{tabular}




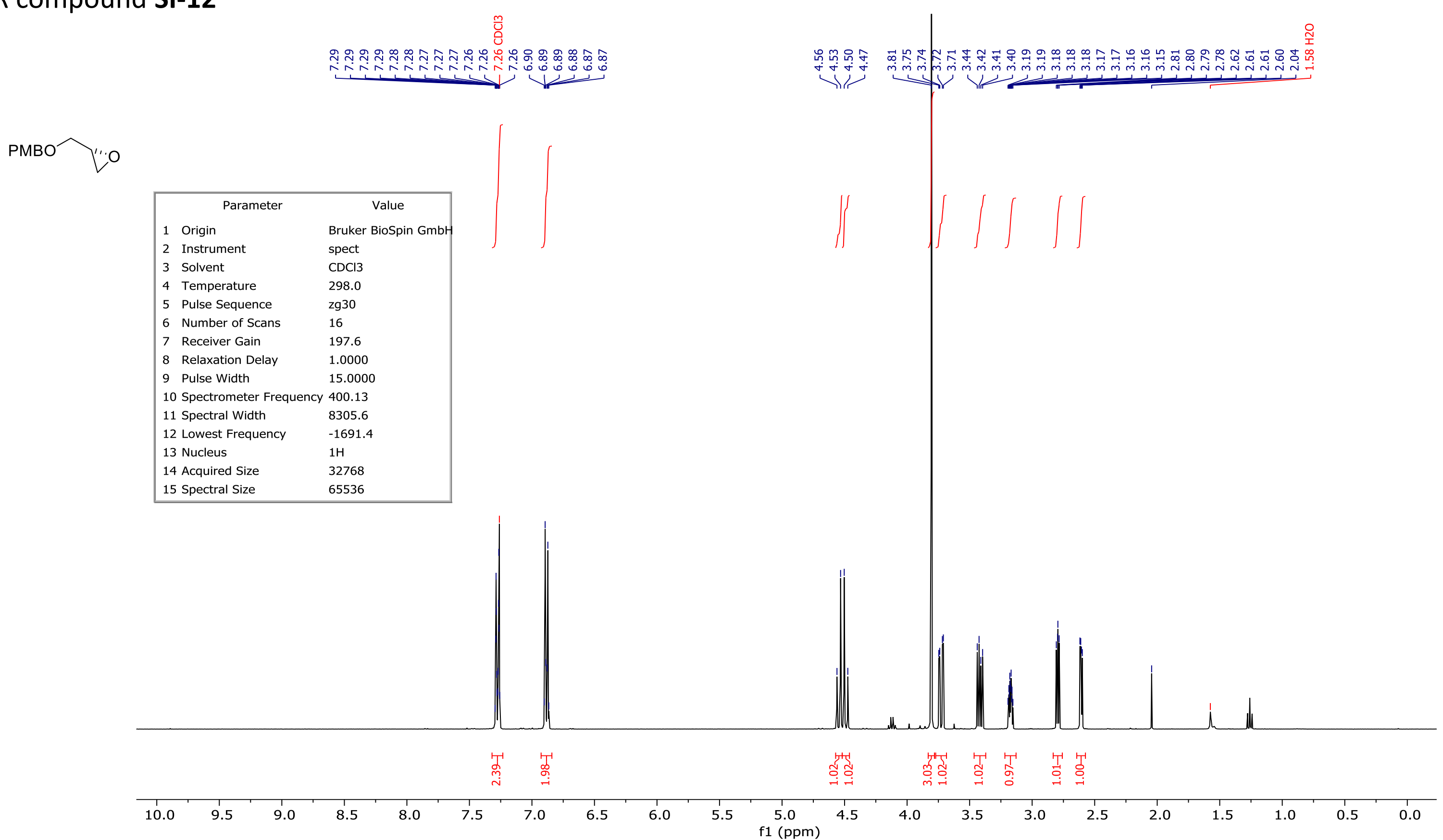




\section{${ }^{13} \mathrm{C}-\mathrm{NMR}$ compound SI-12}

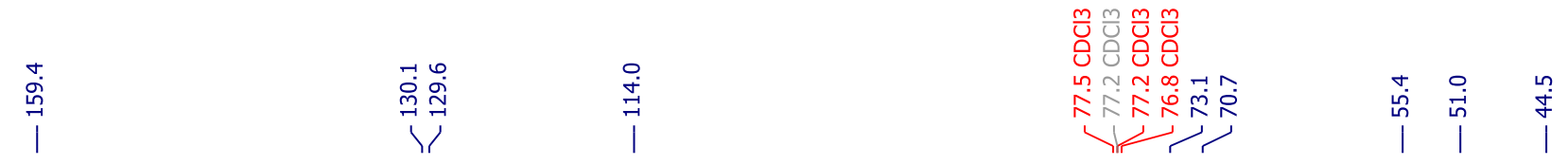

PMBO

\begin{tabular}{|lll|}
\hline \multicolumn{1}{|c|}{ Parameter } & \multicolumn{2}{c|}{ Value } \\
1 & Origin & Bruker BioSpin GmbH \\
2 & Instrument & spect \\
3 & Solvent & CDCl3 \\
4 & Temperature & 298.0 \\
5 & Pulse Sequence & udeft \\
6 & Number of Scans & 256 \\
7 & Receiver Gain & 197.6 \\
8 & Relaxation Delay & 2.0000 \\
9 & Pulse Width & 9.4000 \\
10 & Spectrometer Frequency 100.61 \\
11 & Spectral Width & 26041.7 \\
12 Lowest Frequency & -934.3 \\
13 & Nucleus & $13 \mathrm{C}$ \\
14 & Acquired Size & 4096 \\
15 & Spectral Size & 32768 \\
\hline
\end{tabular}




\section{${ }^{1} \mathrm{H}-\mathrm{NMR}$ compound 9}

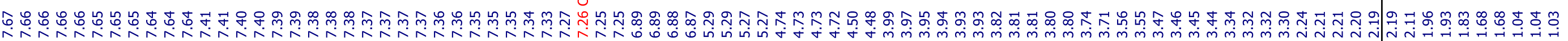
PMBO

OTBDPS
\begin{tabular}{|lll|}
\hline \multicolumn{1}{|c|}{ Parameter } & \multicolumn{2}{c}{ Value } \\
\hline & Origin & \multicolumn{1}{l}{ Bruker BioSpin Gmb } \\
2 & Instrument & spect \\
3 & Solvent & CDCI3 \\
4 & Temperature & 298.0 \\
5 & Pulse Sequence & 2930 \\
6 & Number of Scans & 16 \\
7 & Receiver Gain & 197.6 \\
8 & Relaxation Delay & 1.0000 \\
9 & Pulse Width & 15.0000 \\
10 & Spectrometer Frequency & 400.13 \\
11 & Spectral Width & 8305.6 \\
12 & Lowest Frequency & -1691.7 \\
13 & Nucleus & $1 \mathrm{H}$ \\
14 & Acquired Size & 32768 \\
15 & Spectral Size & 65536 \\
\hline
\end{tabular}
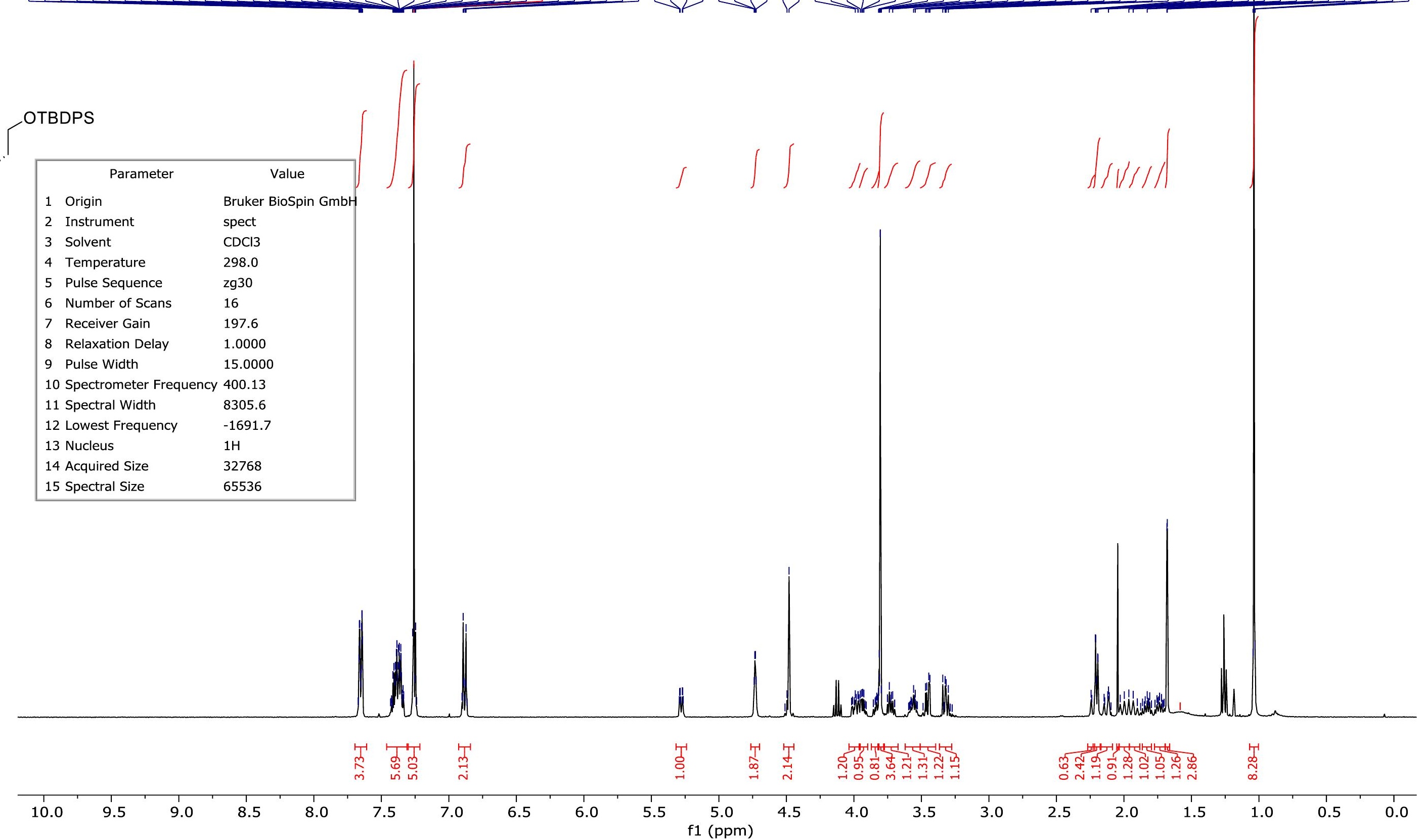


\section{${ }^{13} \mathrm{C}-\mathrm{NMR}$ compound 9}

PMBO

|

"OTBDPS

นก)

\begin{tabular}{|lll|}
\hline \multicolumn{1}{|c|}{ Parameter } & \multicolumn{2}{c|}{ Value } \\
1 & Origin & Bruker BioSpin GmbH \\
2 & Instrument & spect \\
3 & Solvent & CDCI3 \\
4 & Temperature & 298.0 \\
5 & Pulse Sequence & udeft \\
6 & Number of Scans & 1024 \\
7 & Receiver Gain & 197.6 \\
8 & Relaxation Delay & 2.0000 \\
9 & Pulse Width & 9.4000 \\
10 & Spectrometer Frequency 100.61 \\
11 & Spectral Width & 26041.7 \\
12 & Lowest Frequency & -933.0 \\
13 & Nucleus & $13 \mathrm{C}$ \\
14 & Acquired Size & 4096 \\
15 & Spectral Size & 32768 \\
\hline
\end{tabular}

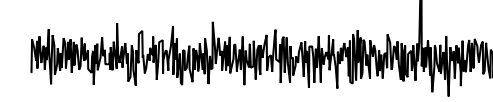

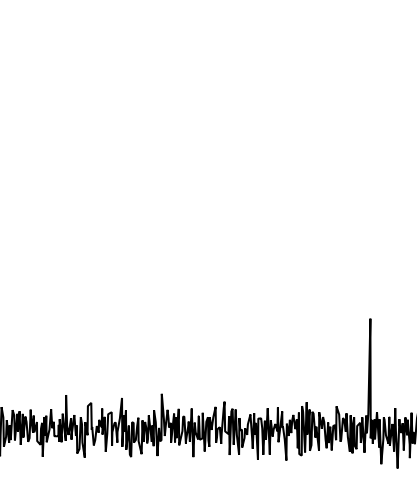




\section{${ }^{1} \mathrm{H}-\mathrm{NMR}$ compound $\mathrm{SI}-13$}

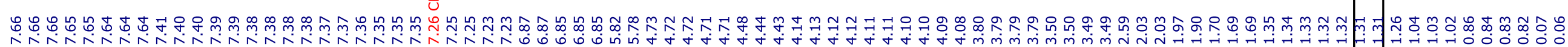
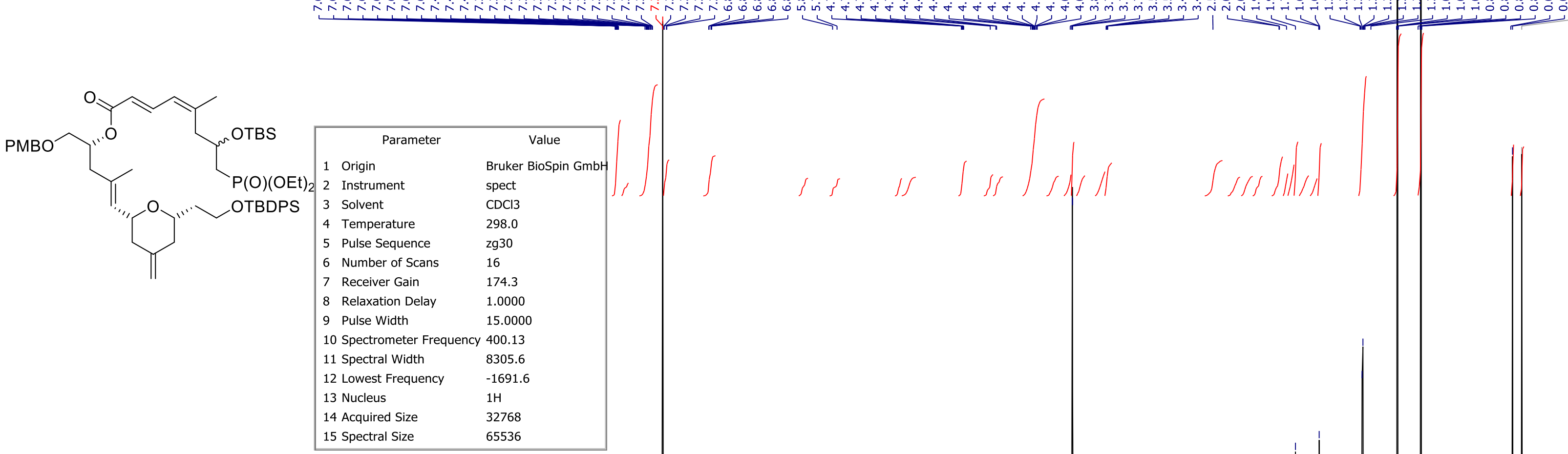


\section{${ }^{13} \mathrm{C}-\mathrm{NMR}$ compound SI-13}

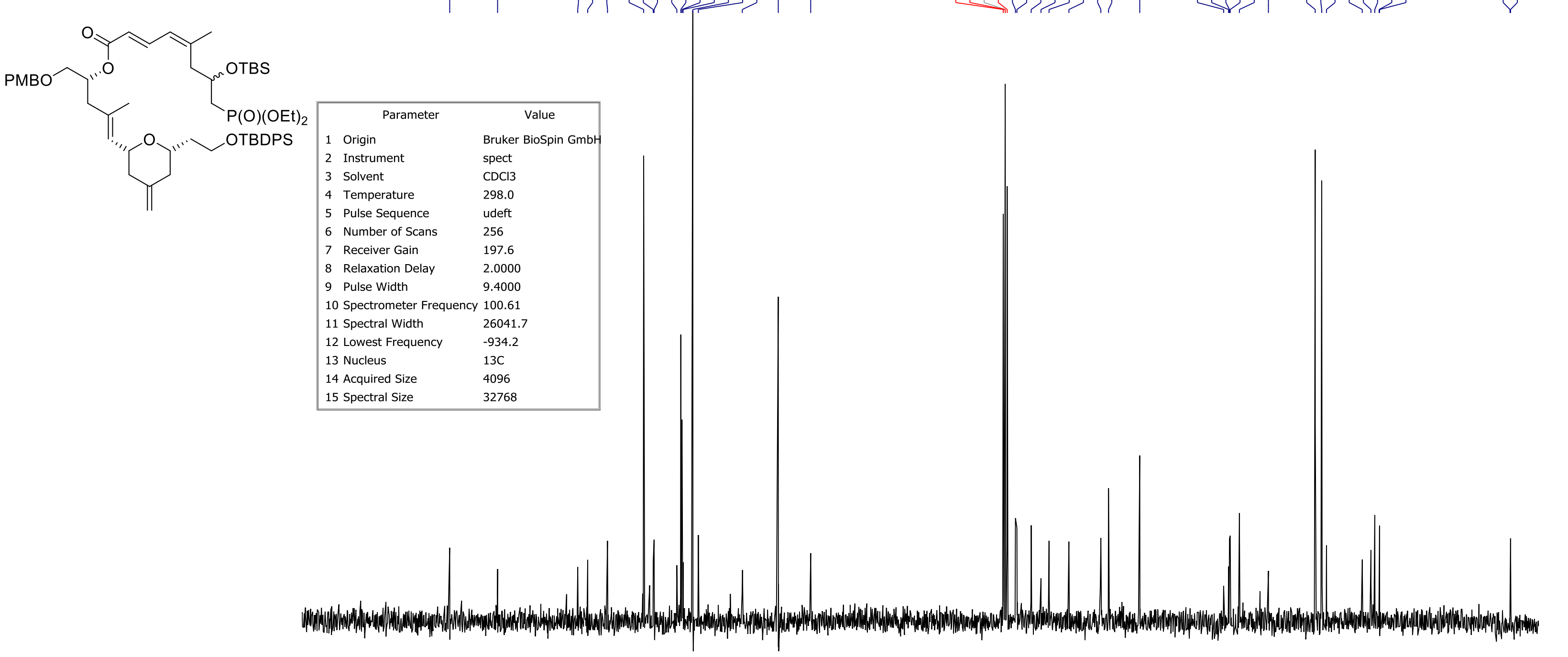

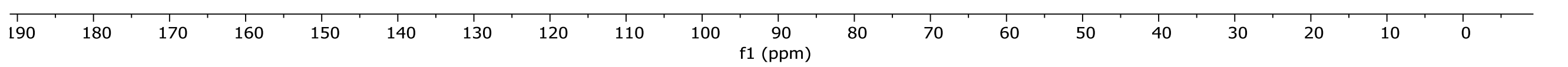




\section{${ }^{1} \mathrm{H}-\mathrm{NMR}$ compound $\mathrm{SI}-14$}

范

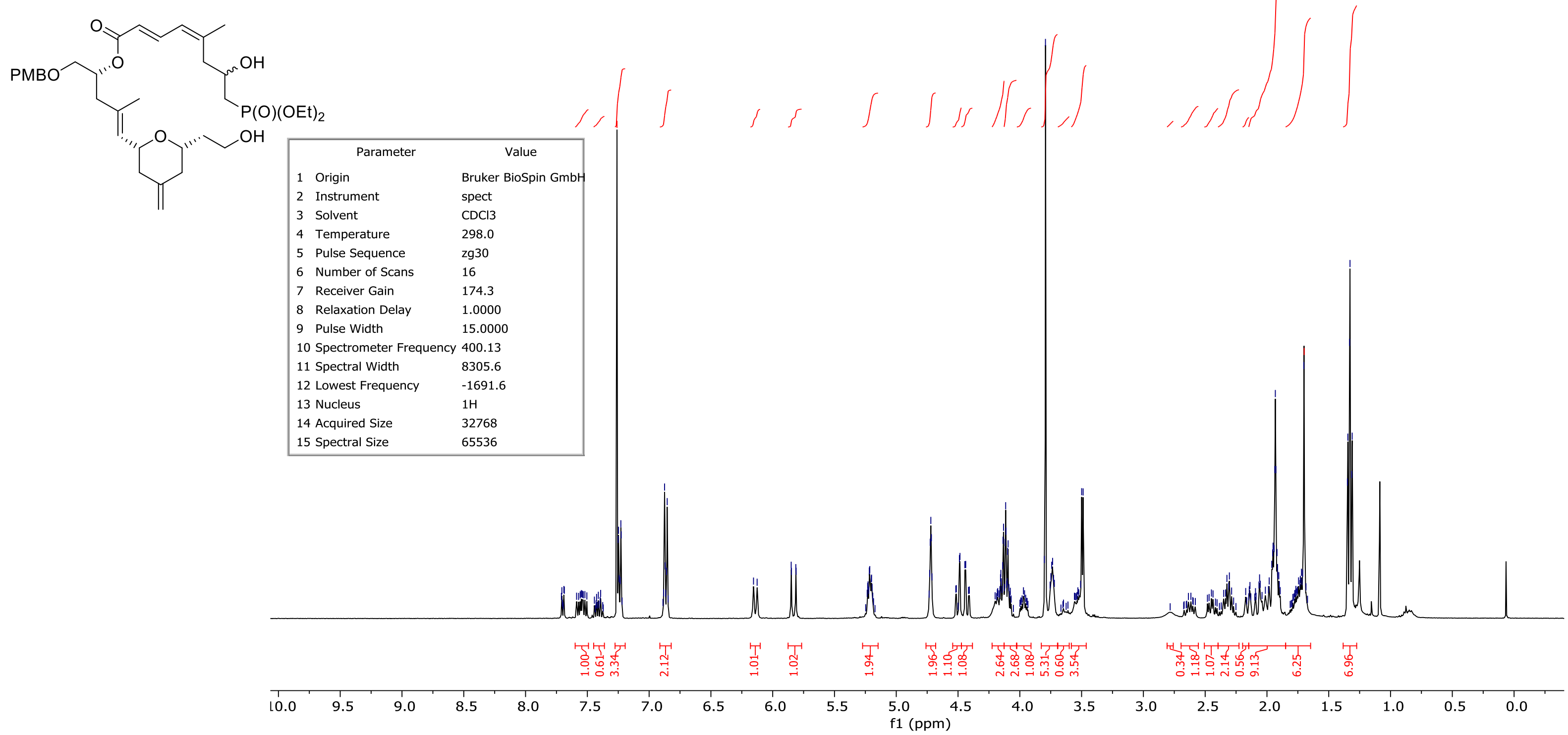




\section{${ }^{13} \mathrm{C}-\mathrm{NMR}$ compound SI-14}

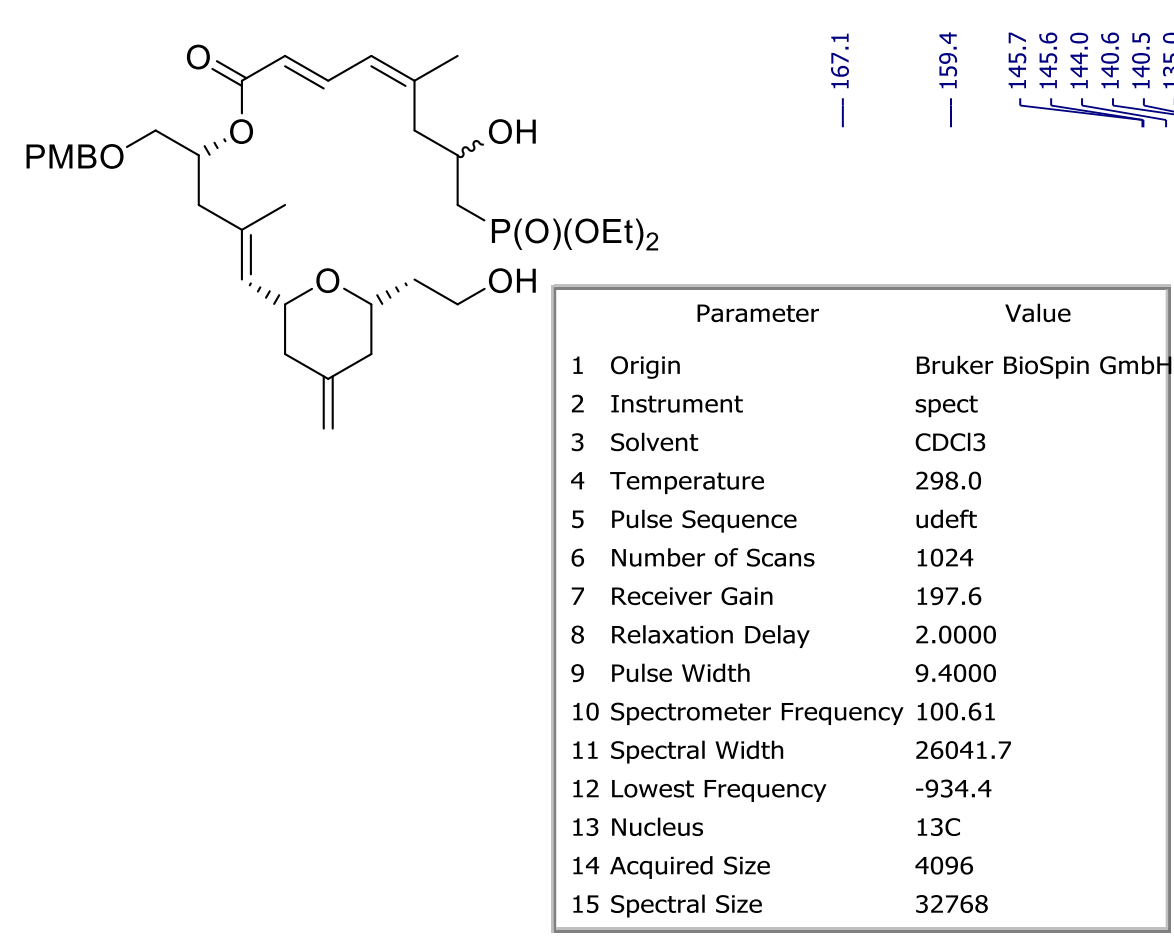

$(\mathrm{O})(\mathrm{OEt})_{2}$

\begin{tabular}{|lll|}
\hline \multicolumn{1}{|c|}{ Parameter } & \multicolumn{1}{c|}{ Value } \\
1 & Origin & Bruker BioSpin GmbH \\
2 & Instrument & spect \\
3 & Solvent & CDCl3 \\
4 & Temperature & 298.0 \\
5 & Pulse Sequence & udeft \\
6 & Number of Scans & 1024 \\
7 & Receiver Gain & 197.6 \\
8 & Relaxation Delay & 2.0000 \\
9 & Pulse Width & 9.4000 \\
10 & Spectrometer Frequency 100.61 \\
11 & Spectral Width & 26041.7 \\
12 & Lowest Frequency & -934.4 \\
13 & Nucleus & $13 C$ \\
14 & Acquired Size & 4096 \\
15 & Spectral Size & 32768 \\
\hline
\end{tabular}

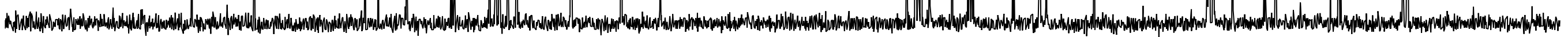

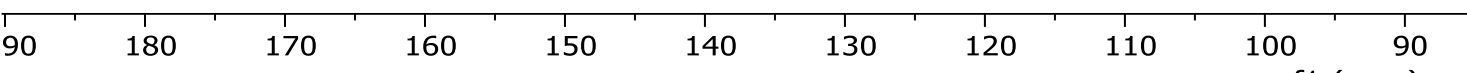

$80 \quad 70 \quad 60$

60

40

30

30




\section{${ }^{1} \mathrm{H}-\mathrm{NMR}$ compound $\mathrm{SI}-15$}

莒

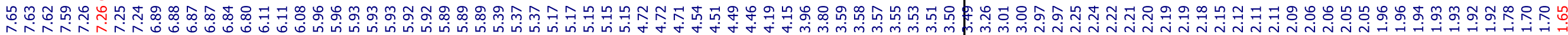

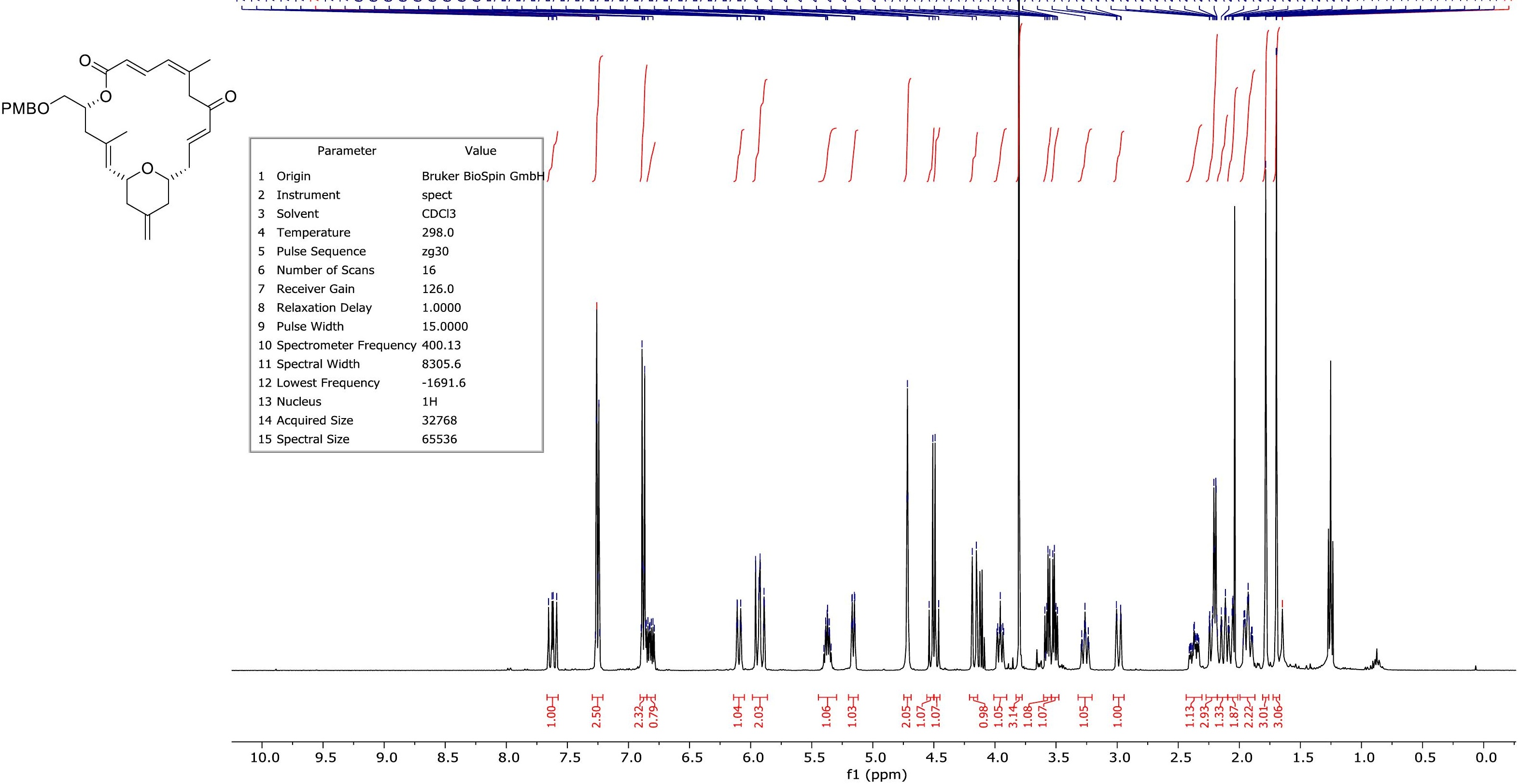




\section{${ }^{13} \mathrm{C}-\mathrm{NMR}$ compound SI-15}

|
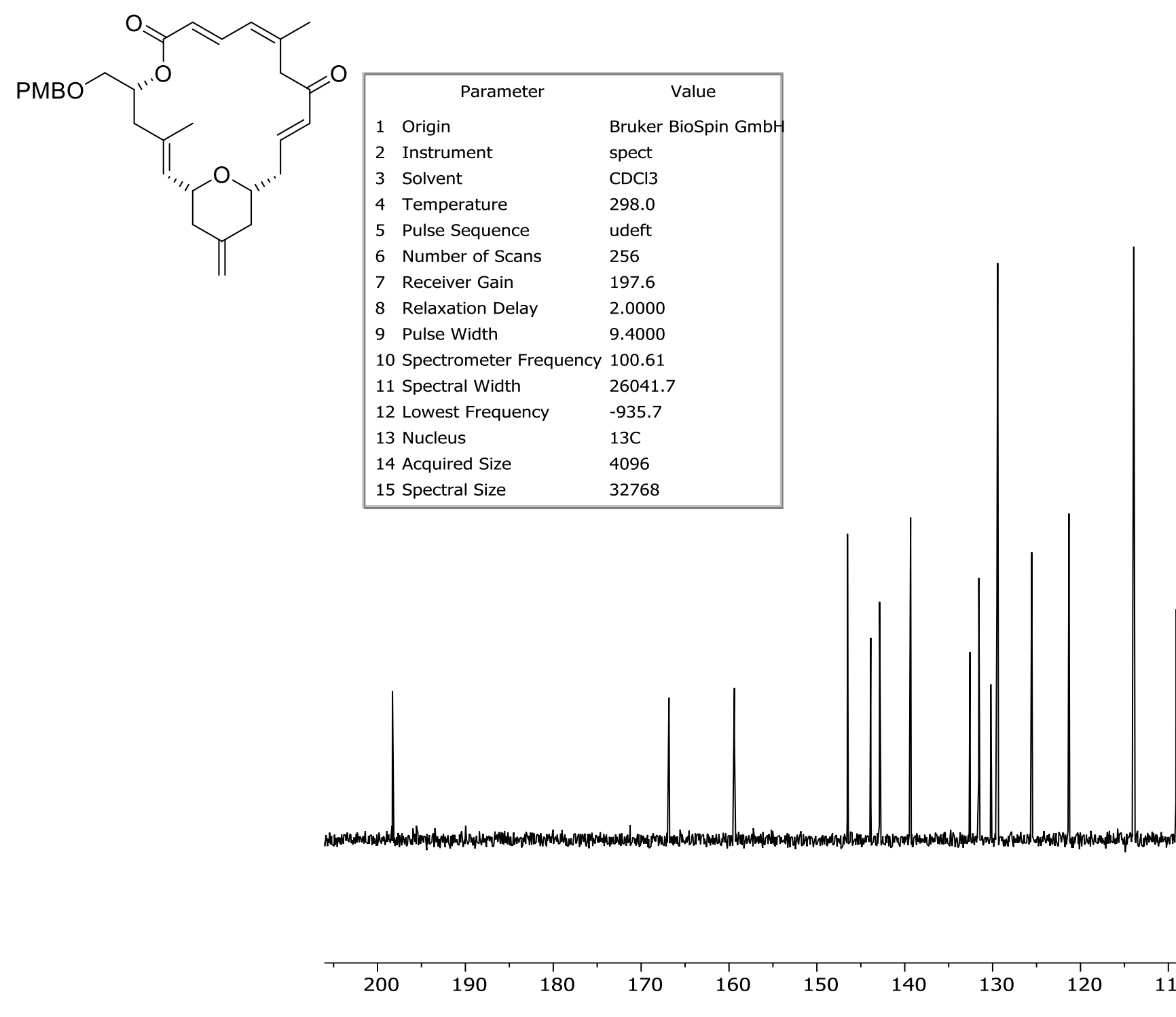

100
$f 1(p p m)$

90

80

70

60

50

40

30 


\section{${ }^{1} \mathrm{H}-\mathrm{NMR}$ compound SI-16}

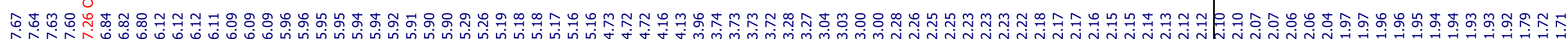
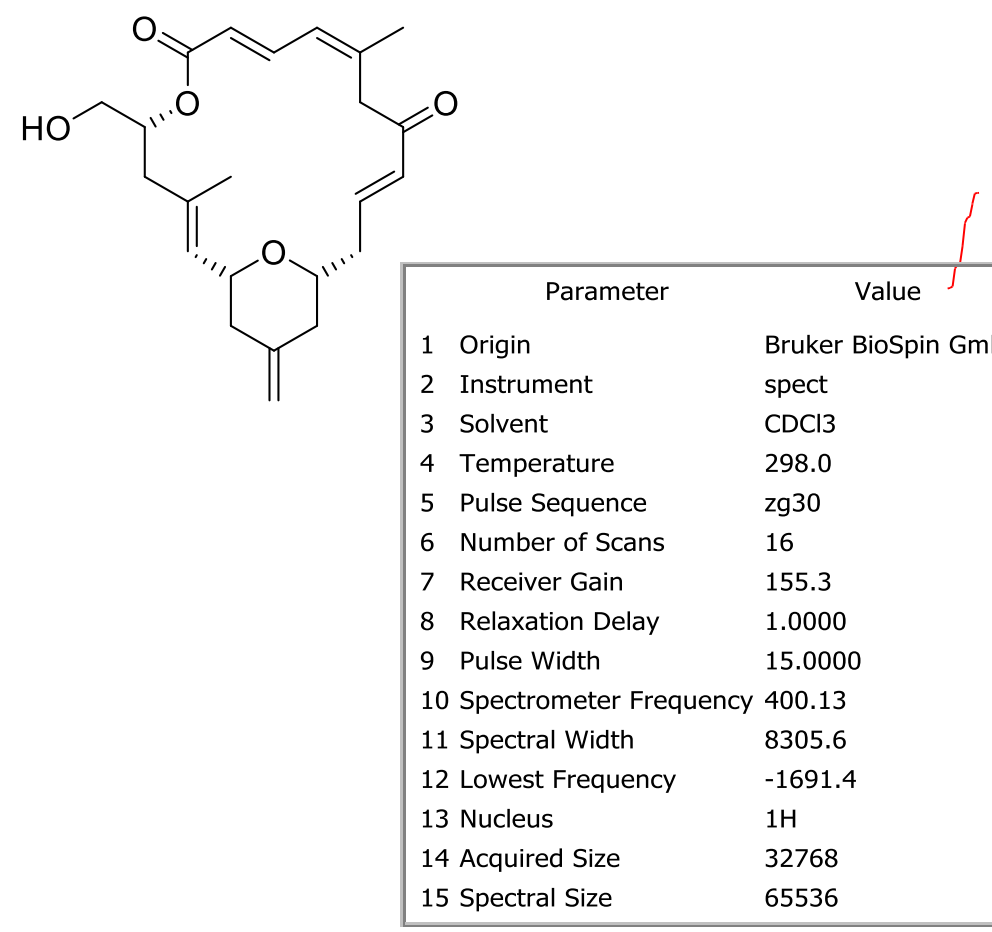

H
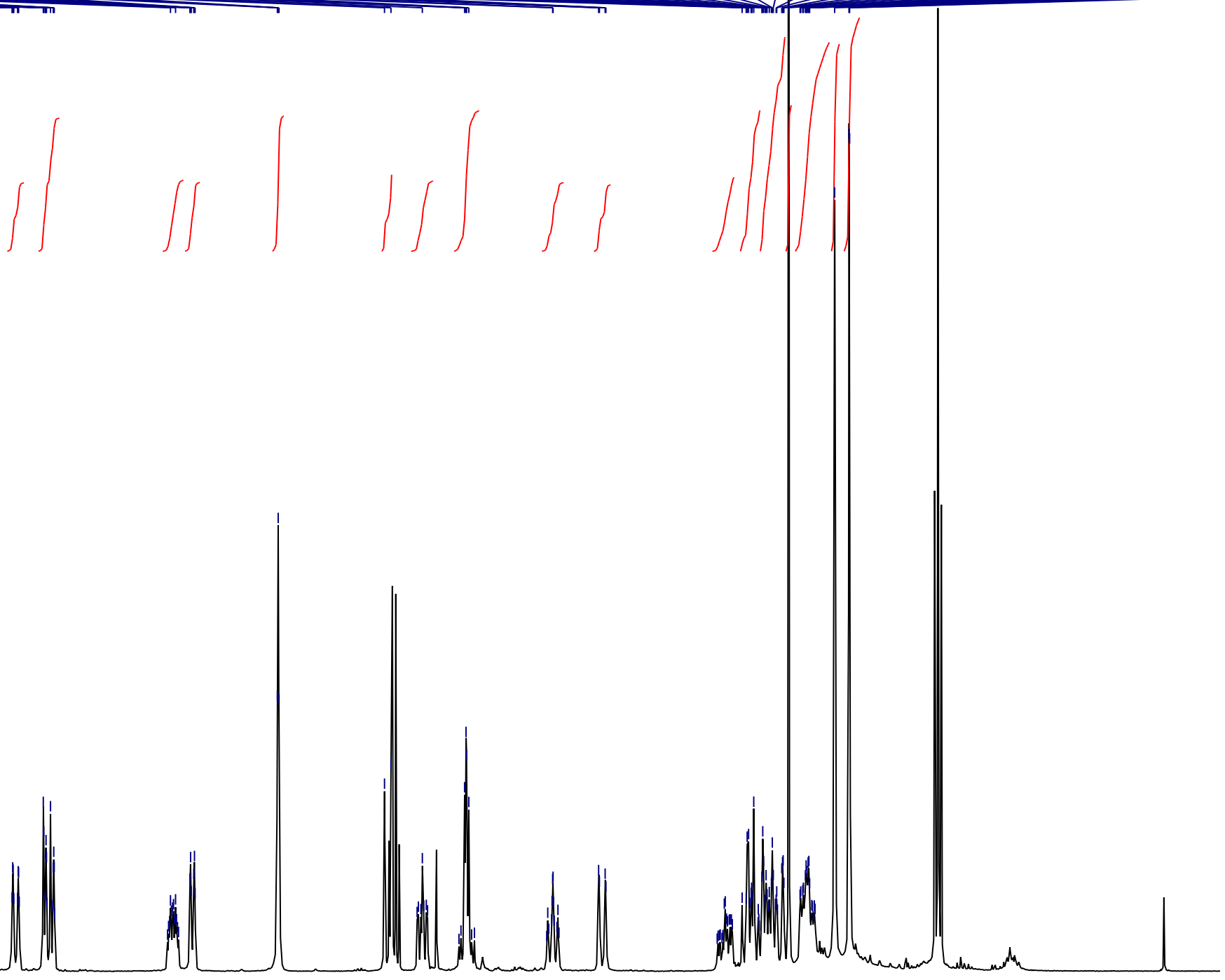

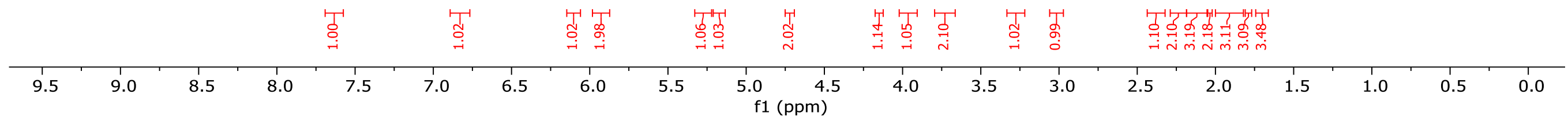




\section{${ }^{13} \mathrm{C}-\mathrm{NMR}$ compound SI-16}

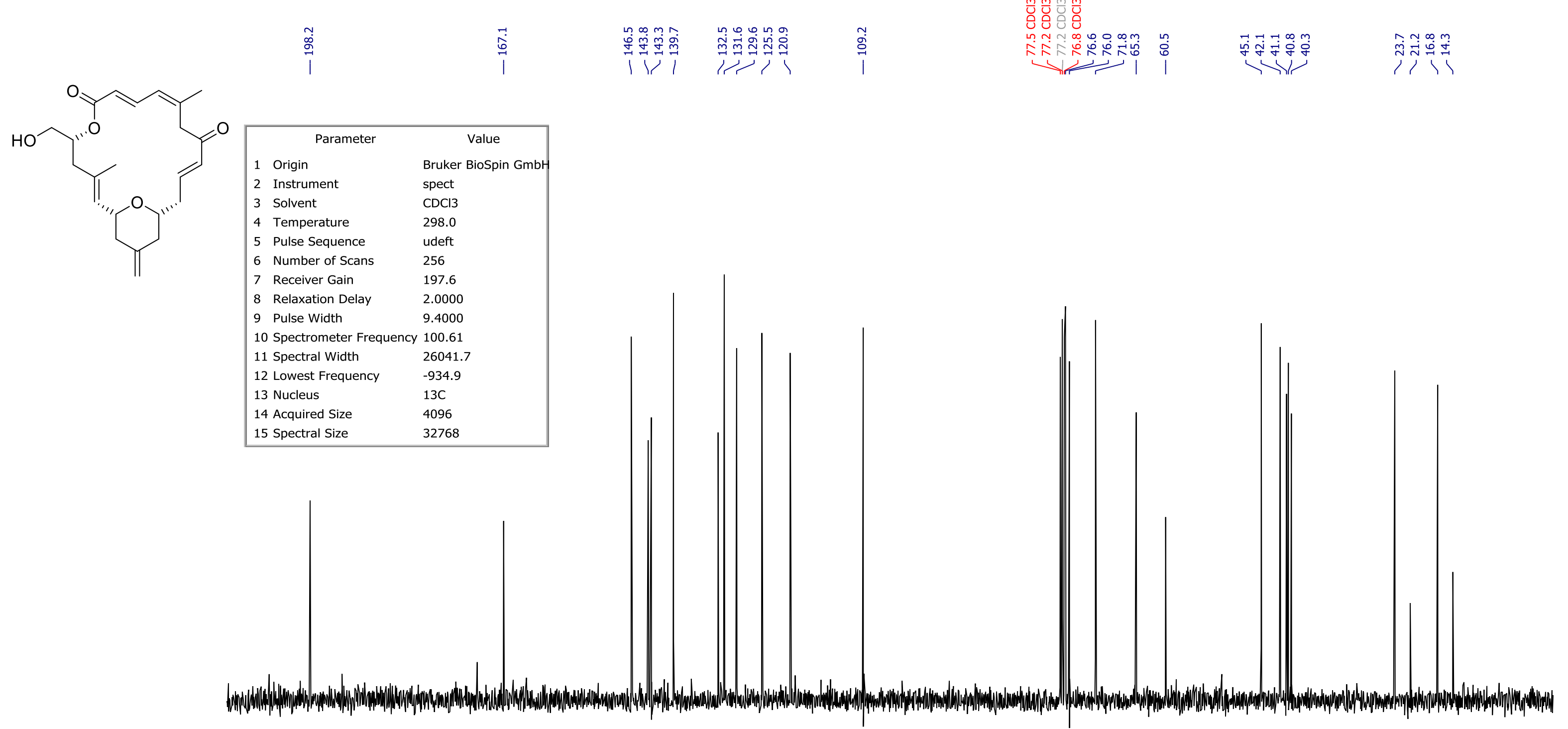

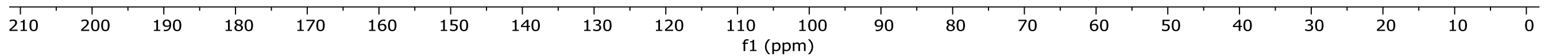




\section{${ }^{1} \mathrm{H}-\mathrm{NMR}$ compound ent-2}

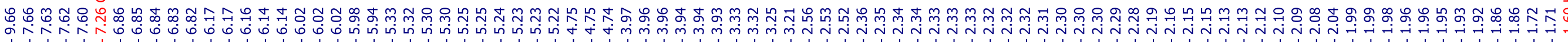

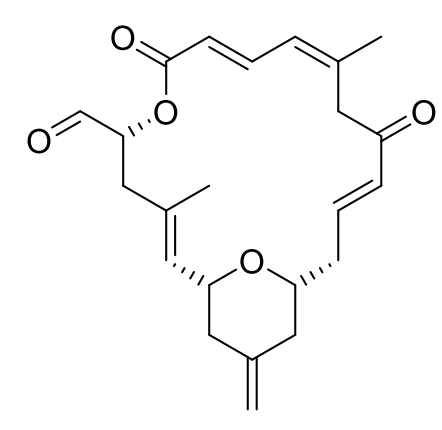

\begin{tabular}{|lll|}
\hline \multicolumn{1}{|c|}{ Parameter } & \multicolumn{1}{c|}{ Value } \\
1 & Origin & Bruker BioSpin GmbH \\
2 & Instrument & spect \\
3 & Solvent & $\mathrm{CDCl3}$ \\
4 & Temperature & 298.0 \\
5 & Pulse Sequence & $\mathrm{zg} 30$ \\
6 & Number of Scans & 16 \\
7 & Receiver Gain & 197.6 \\
8 & Relaxation Delay & 1.0000 \\
9 & Pulse Width & 15.0000 \\
10 & Spectrometer Frequency & 400.13 \\
11 & Spectral Width & 8305.6 \\
12 & Lowest Frequency & -1691.4 \\
13 Nucleus & $1 \mathrm{H}$ \\
14 Acquired Size & 32768 \\
15 & Spectral Size & 65536 \\
\hline
\end{tabular}
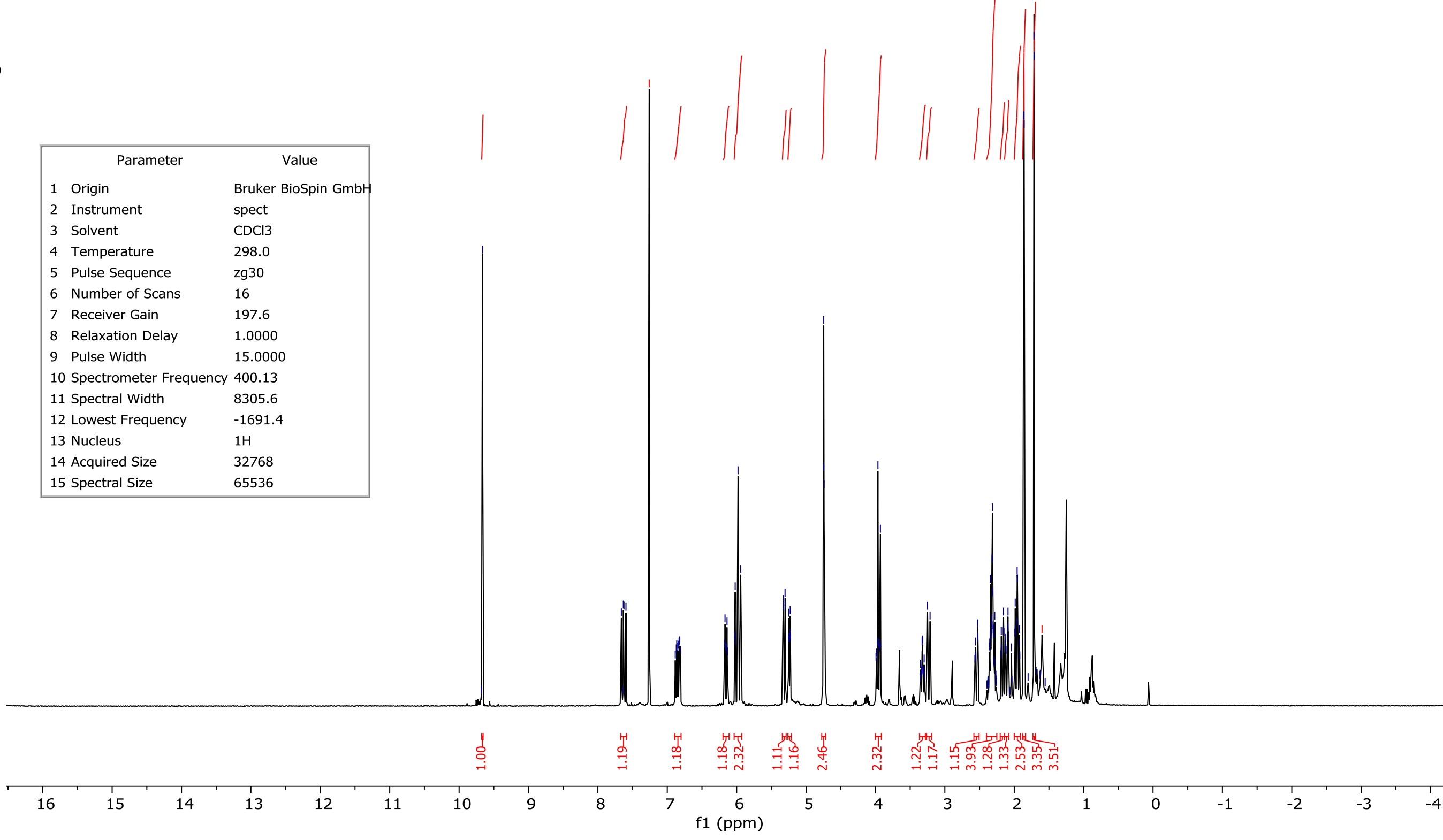


\section{${ }^{13} \mathrm{C}-\mathrm{NMR}$ compound ent-2}

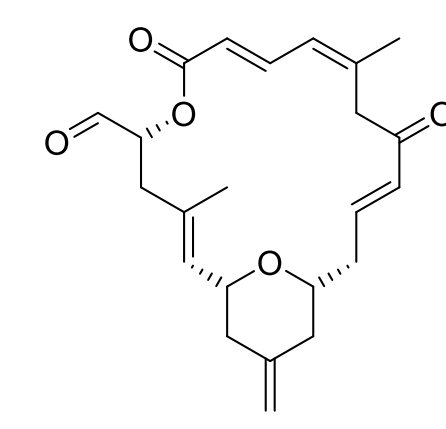

$$
\text { ํํำ }
$$

\begin{tabular}{|lll|}
\hline \multicolumn{1}{|c|}{ Parameter } & \multicolumn{2}{c|}{ Value } \\
1 & Origin & Bruker BioSpin GmbH \\
2 & Instrument & spect \\
3 & Solvent & CDCl3 \\
4 & Temperature & 298.0 \\
5 & Pulse Sequence & udeft \\
6 & Number of Scans & 256 \\
7 & Receiver Gain & 197.6 \\
8 & Relaxation Delay & 2.0000 \\
9 & Pulse Width & 9.4000 \\
10 & Spectrometer Frequency 100.61 \\
11 & Spectral Width & 26041.7 \\
12 & Lowest Frequency & -934.2 \\
13 & Nucleus & $13 C$ \\
14 & Acquired Size & 4096 \\
15 & Spectral Size & 32768 \\
\hline
\end{tabular}

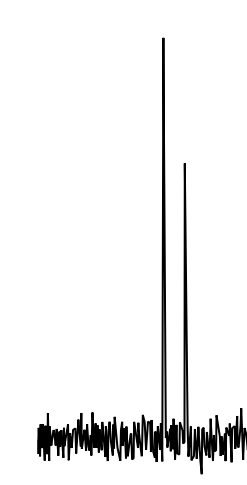




\section{${ }^{1} \mathrm{H}-\mathrm{NMR}$ compound ent-1}

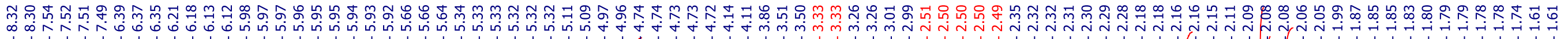
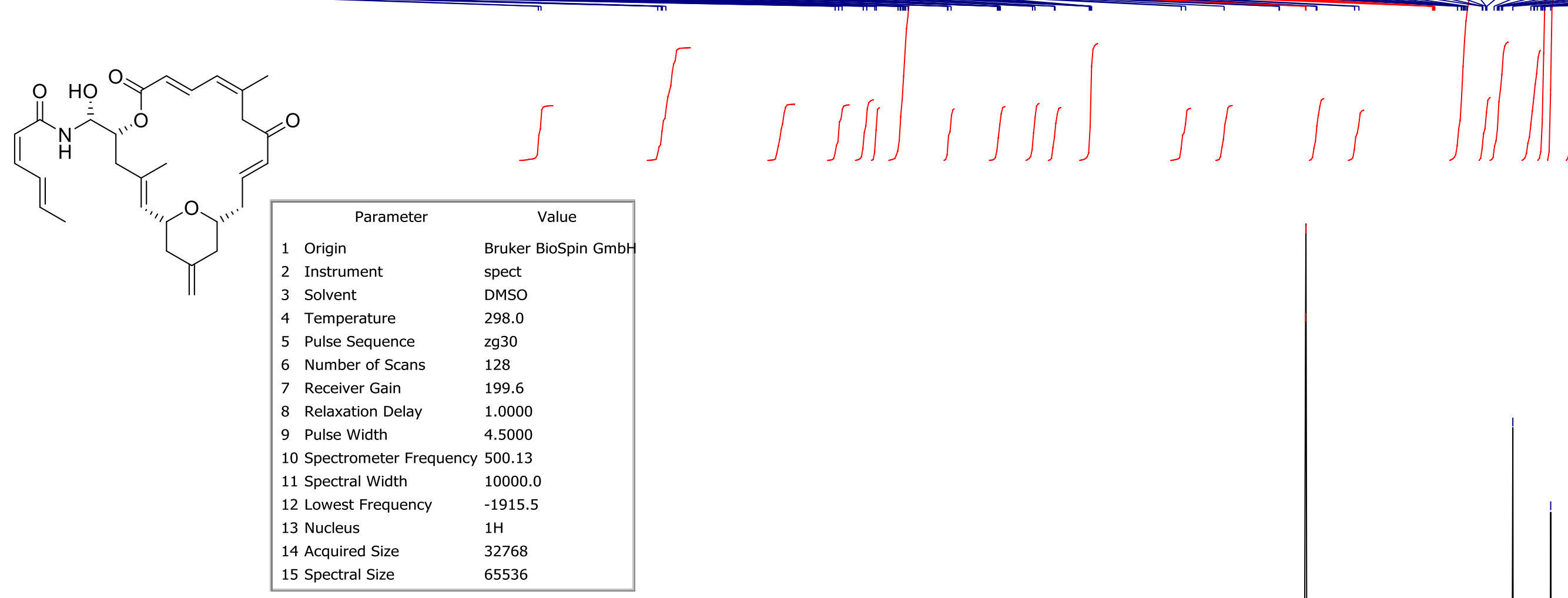

\begin{tabular}{|lll|}
\multicolumn{1}{c|}{ Parameter } & \multicolumn{1}{c|}{ Value } \\
1 & Origin & Bruker BioSpin GmbH \\
2 & Instrument & spect \\
3 & Solvent & DMSO \\
4 & Temperature & 298.0 \\
5 & Pulse Sequence & zg30 \\
6 & Number of Scans & 128 \\
7 & Receiver Gain & 199.6 \\
8 & Relaxation Delay & 1.0000 \\
9 & Pulse Width & 4.5000 \\
10 & Spectrometer Frequency & 500.13 \\
11 & Spectral Width & 10000.0 \\
12 & Lowest Frequency & -1915.5 \\
13 & Nucleus & $1 \mathrm{H}$ \\
14 & Acquired Size & 32768 \\
15 & Spectral Size & 65536 \\
\hline
\end{tabular}

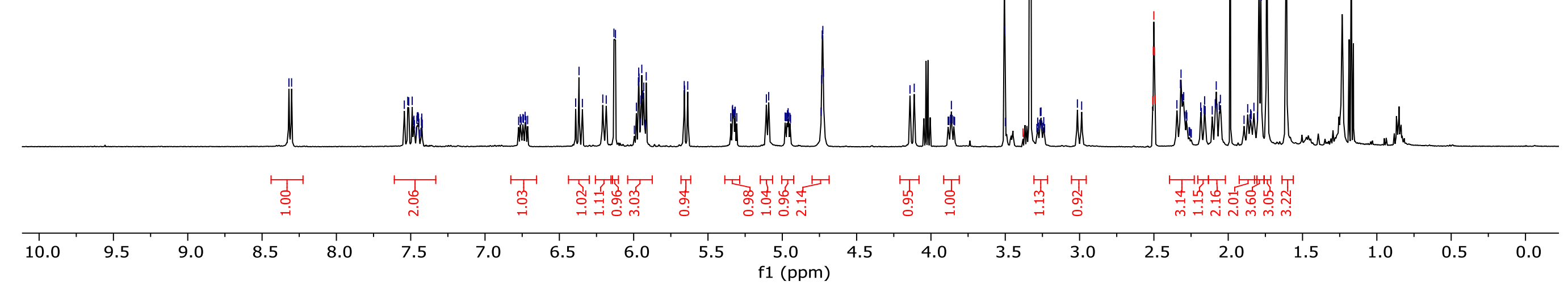




\section{${ }^{13} \mathrm{C}-\mathrm{NMR}$ compound ent-1}

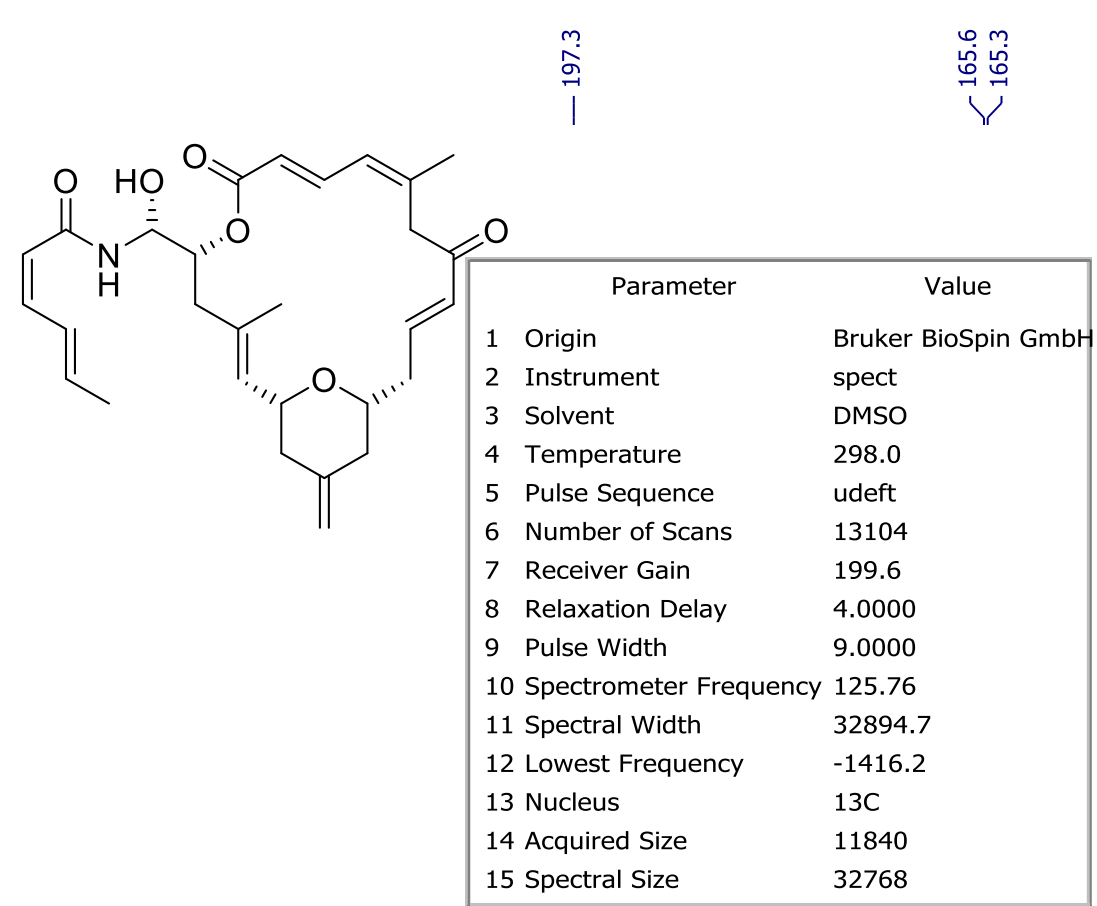




\section{${ }^{1} \mathrm{H}-\mathrm{NMR}$ compound $\mathbf{S I - 1 7}$}

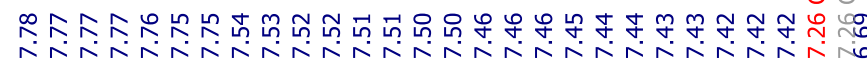
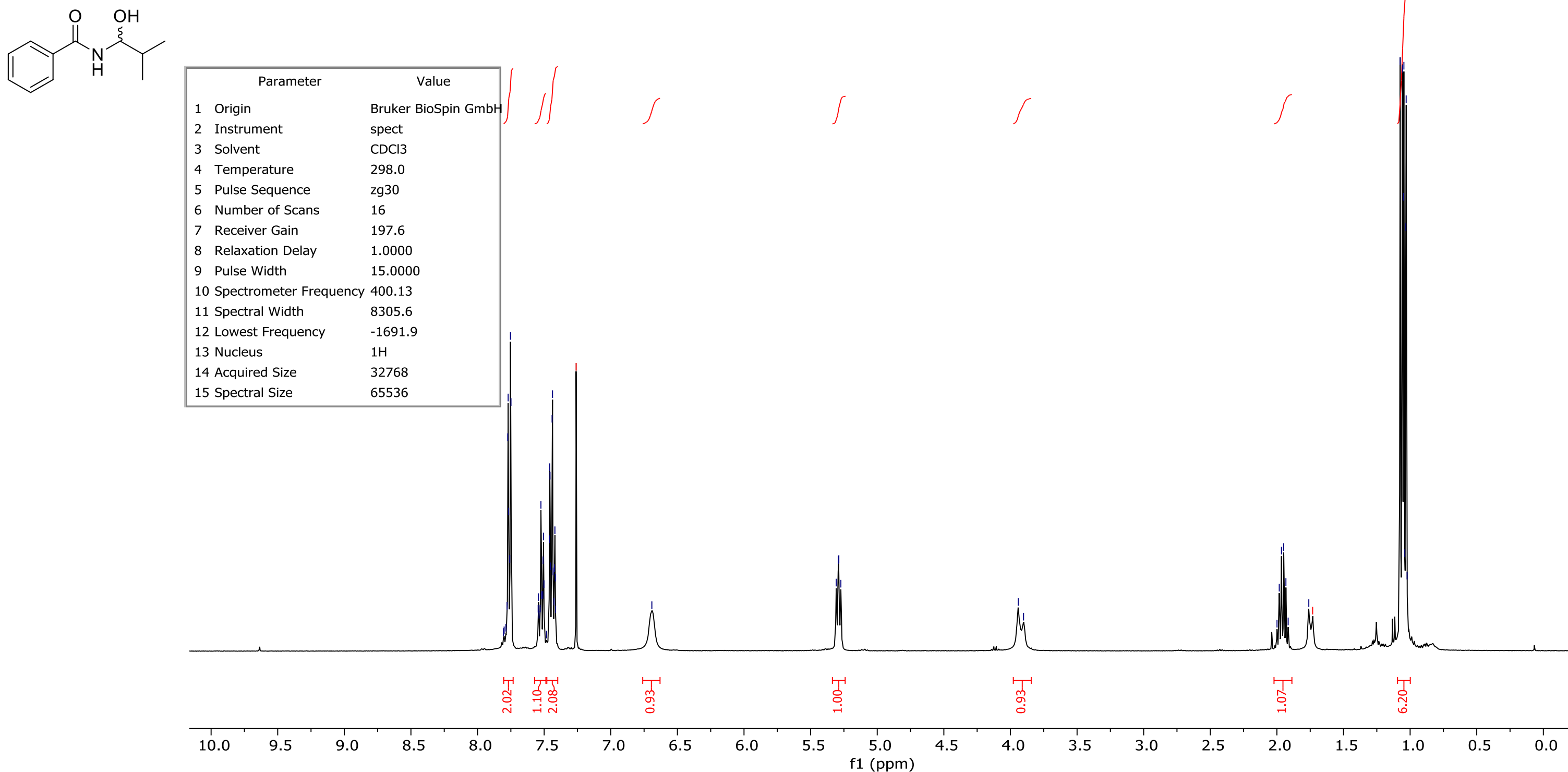


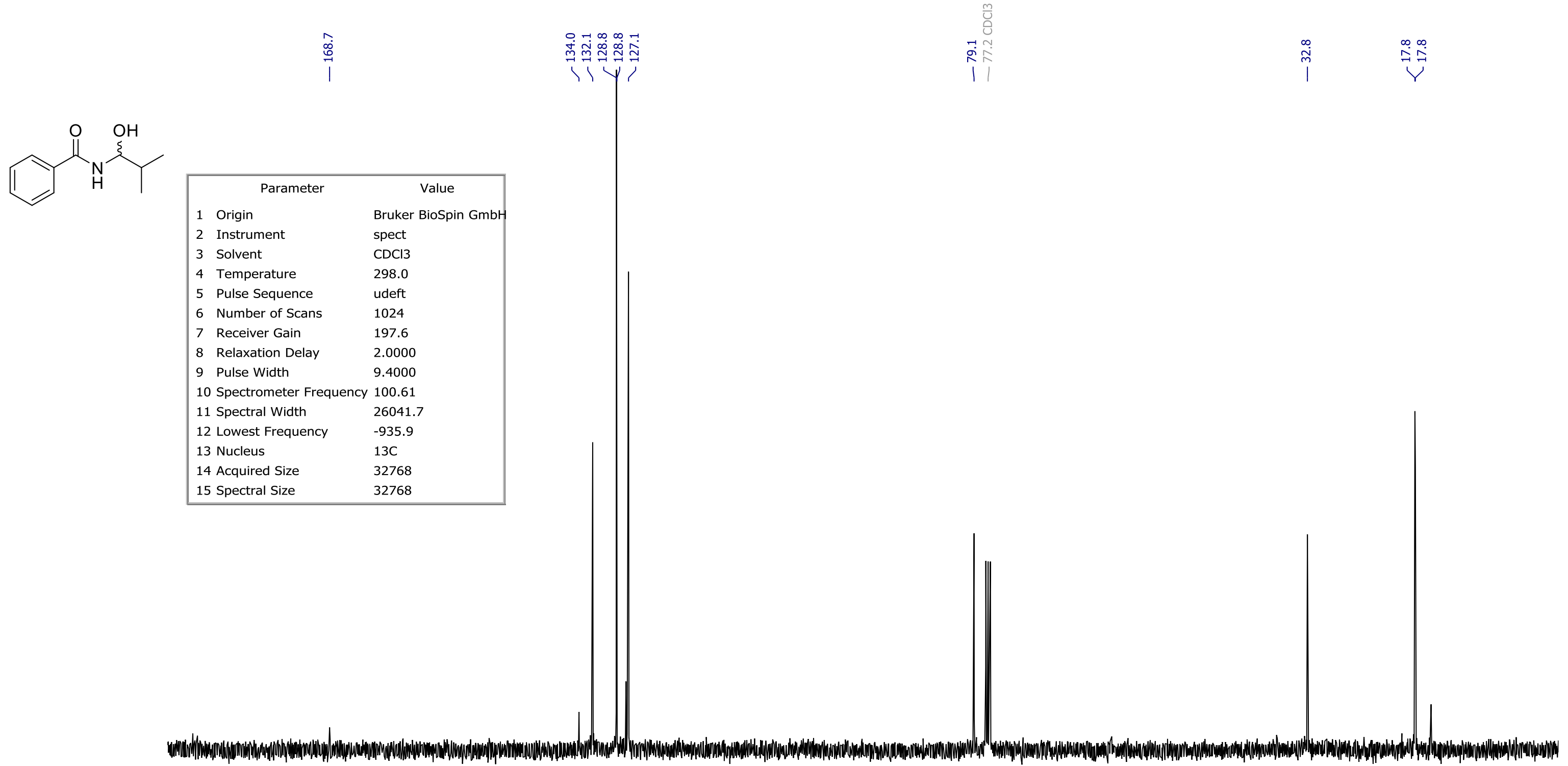




\section{${ }^{1} \mathrm{H}-\mathrm{NMR}$ compound $\mathrm{SI}-\mathbf{1 8}$}

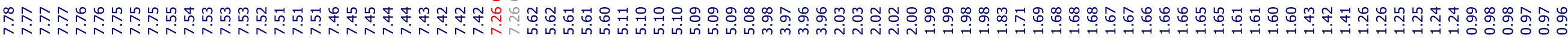

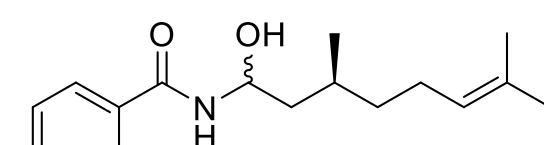

\begin{tabular}{|lll|}
\hline \multicolumn{1}{|c|}{ Parameter } & \multicolumn{1}{c|}{ Value } \\
1 & Origin & Bruker BioSpin GmbH \\
2 & Instrument & spect \\
3 & Solvent & CDCl3 \\
4 & Temperature & 298.0 \\
5 & Pulse Sequence & $\mathrm{zg} 30$ \\
6 & Number of Scans & 16 \\
7 & Receiver Gain & 111.0 \\
8 & Relaxation Delay & 1.0000 \\
9 & Pulse Width & 15.0000 \\
10 & Spectrometer Frequency & 400.13 \\
11 & Spectral Width & 8305.6 \\
12 & Lowest Frequency & -1691.5 \\
13 & Nucleus & $1 \mathrm{H}$ \\
14 & Acquired Size & 32768 \\
15 & Spectral Size & 65536 \\
\hline
\end{tabular}

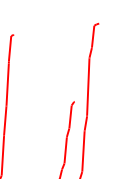
65536

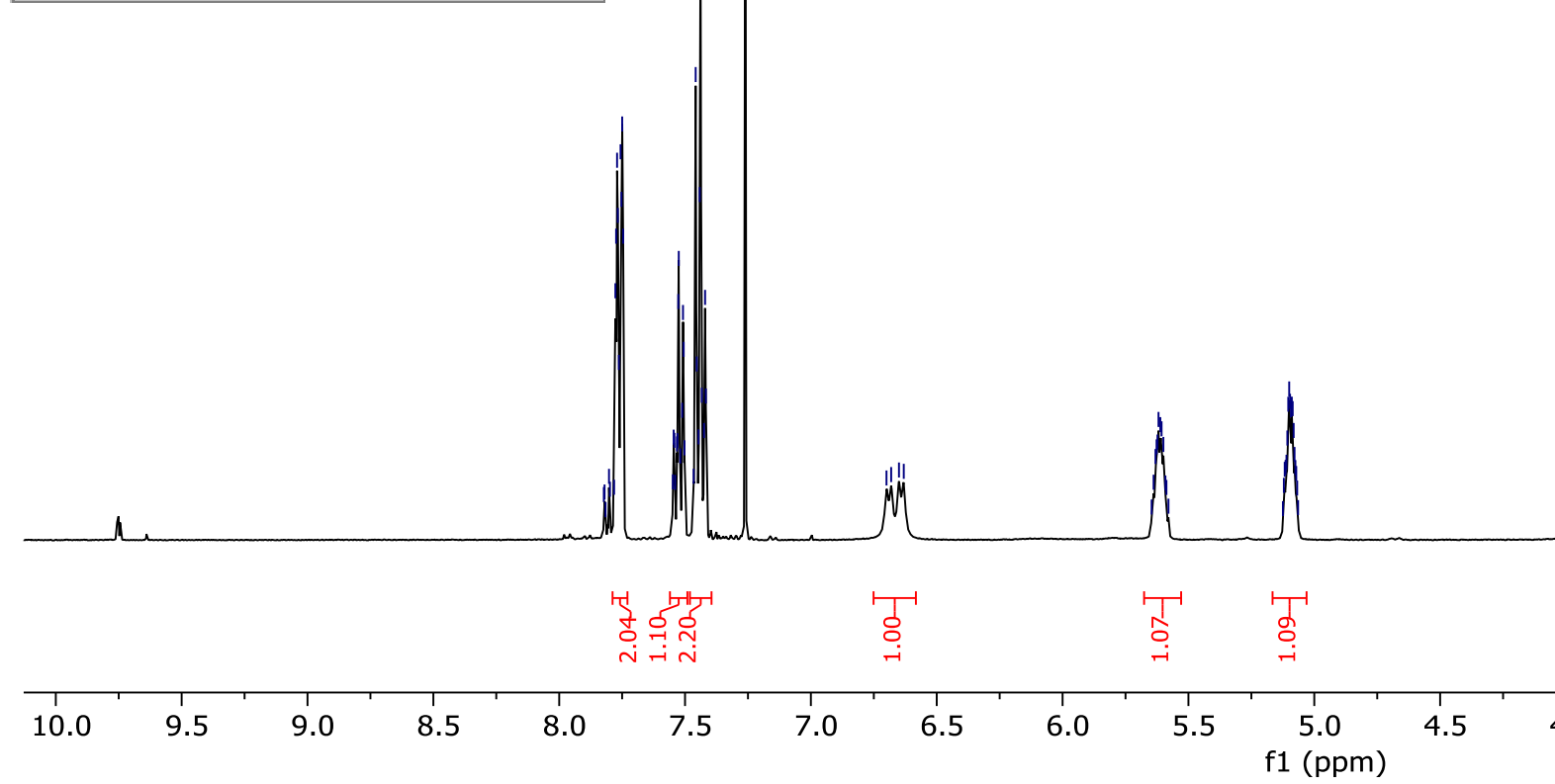




\section{${ }^{13} \mathrm{C}-\mathrm{NMR}$ compound $\mathrm{SI-18}$}

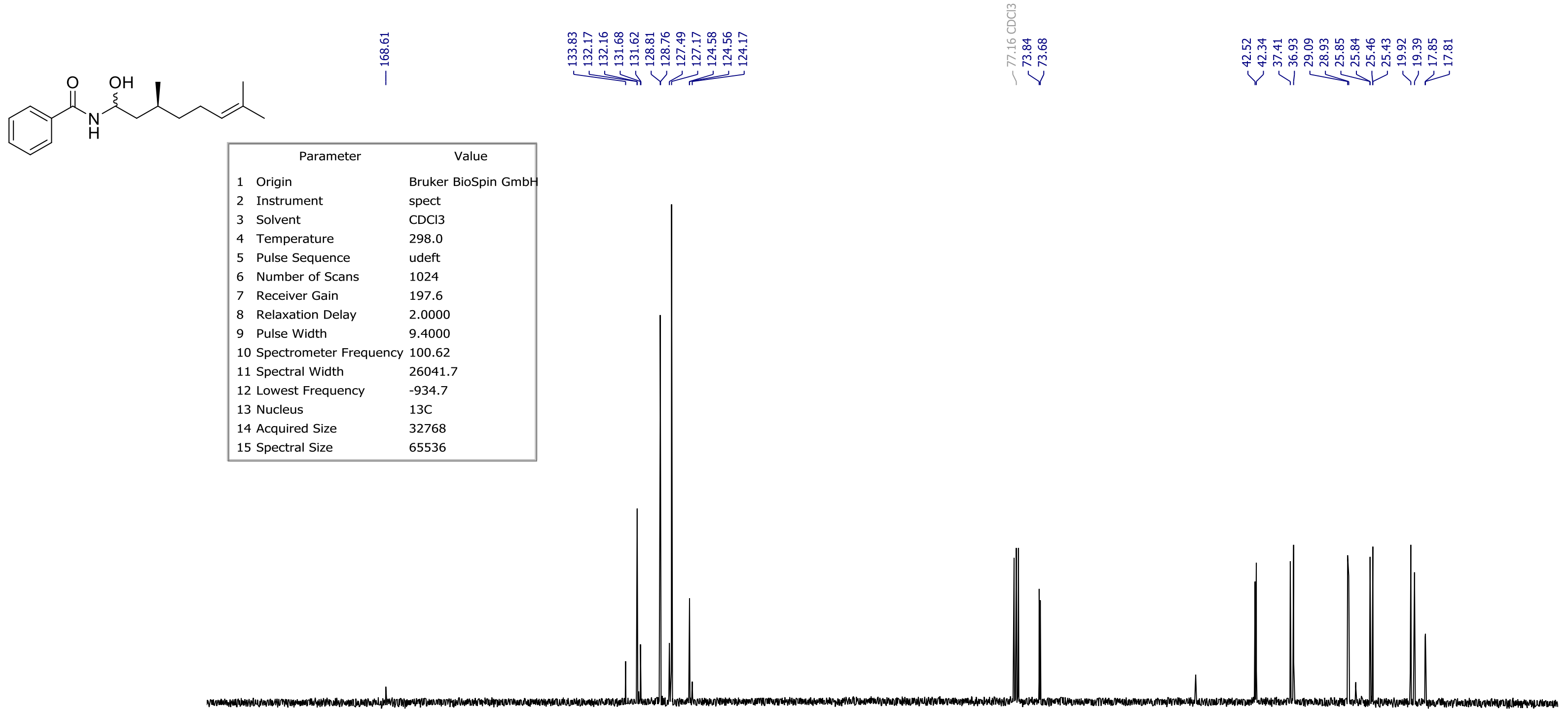




\section{${ }^{1} \mathrm{H}-\mathrm{NMR}$ compound 13}

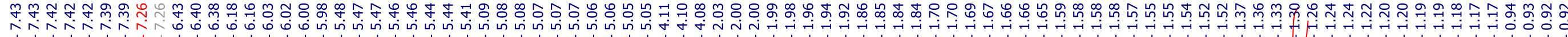

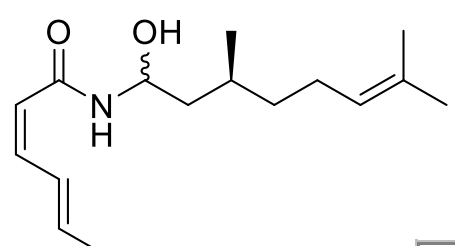

\begin{tabular}{|lll|}
\hline \multicolumn{1}{|c|}{ Parameter } & \multicolumn{1}{c|}{ Value } \\
1 & Origin & Bruker BioSpin GmbH \\
2 & Instrument & spect \\
3 & Solvent & CDCl3 \\
4 & Temperature & 298.0 \\
5 & Pulse Sequence & $\mathrm{zg} 30$ \\
6 & Number of Scans & 16 \\
7 & Receiver Gain & 49.5 \\
8 & Relaxation Delay & 1.0000 \\
9 & Pulse Width & 15.0000 \\
10 & Spectrometer Frequency & 400.13 \\
11 & Spectral Width & 8305.6 \\
12 & Lowest Frequency & -1691.5 \\
13 & Nucleus & $1 \mathrm{H}$ \\
14 & Acquired Size & 32768 \\
15 & Spectral Size & 32768 \\
\hline
\end{tabular}
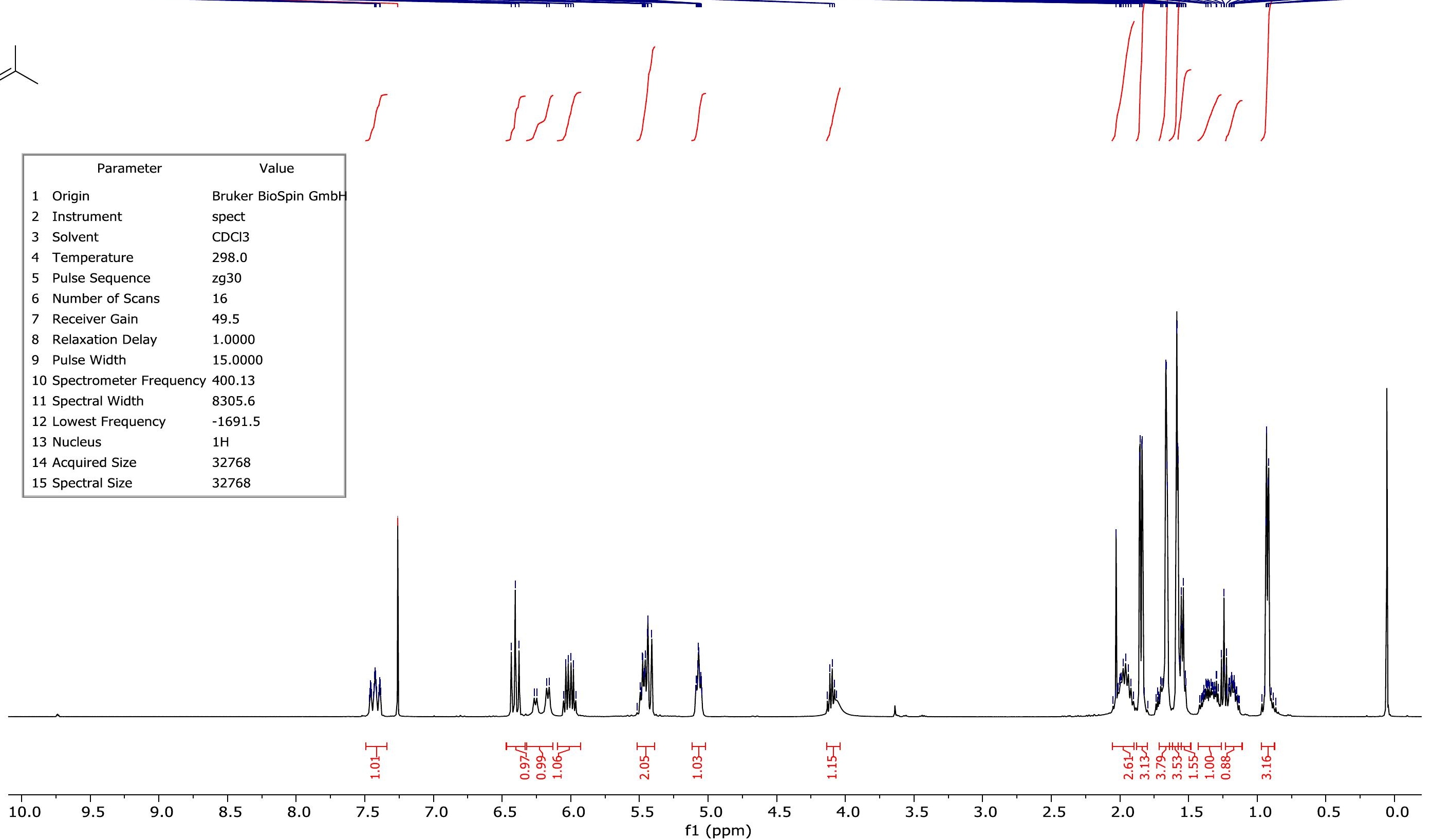


\section{${ }^{13} \mathrm{C}-\mathrm{NMR}$ compound 13}

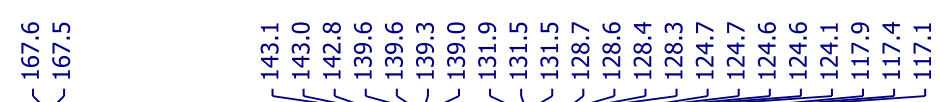

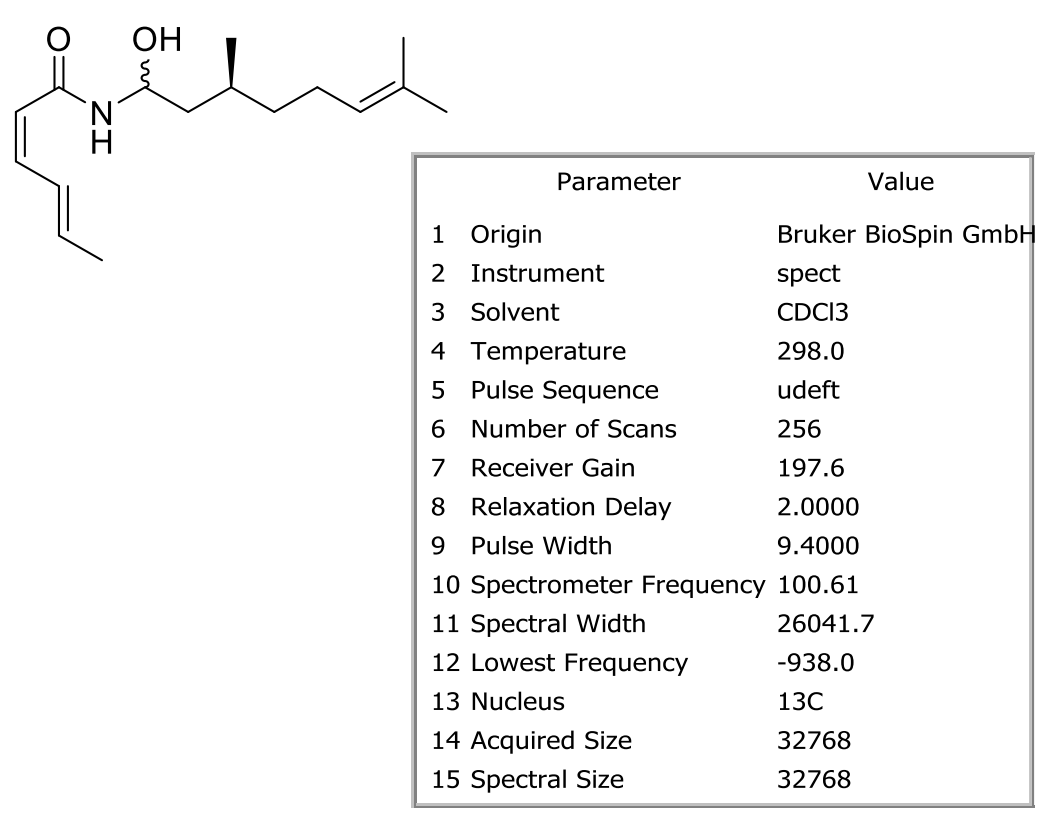

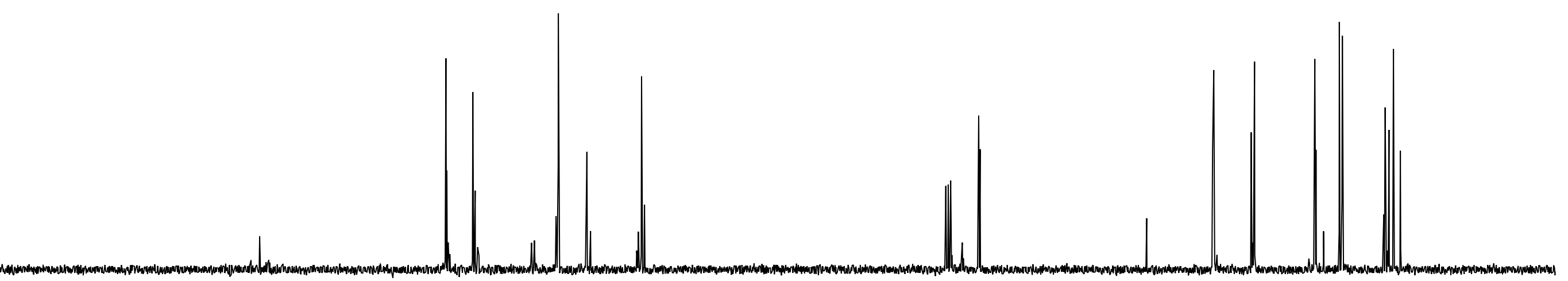




\section{${ }^{1} \mathrm{H}-\mathrm{NMR}$ compound SI-20}

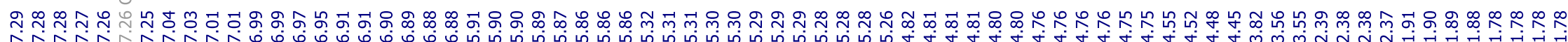
$\overbrace{}^{\text {PMBO }}$

\begin{tabular}{lll|}
\hline \multicolumn{1}{c}{ Parameter } & \multicolumn{1}{c}{ Value } \\
1 & Origin & Bruker BioSpin GmbF \\
2 & Instrument & spect \\
3 & Solvent & $\mathrm{CDCl} 3$ \\
4 & Temperature & 298.0 \\
5 & Pulse Sequence & $\mathrm{zg} 30$ \\
6 & Number of Scans & 16 \\
7 & Receiver Gain & 30.0 \\
8 & Relaxation Delay & 1.0000 \\
9 & Pulse Width & 15.0000 \\
10 & Spectrometer Frequency & 400.13 \\
11 & Spectral Width & 8305.6 \\
12 & Lowest Frequency & -1678.7 \\
13 & Nucleus & $1 \mathrm{H}$ \\
14 & Acquired Size & 32768 \\
15 & Spectral Size & 65536 \\
\hline \multicolumn{2}{l}{}
\end{tabular}
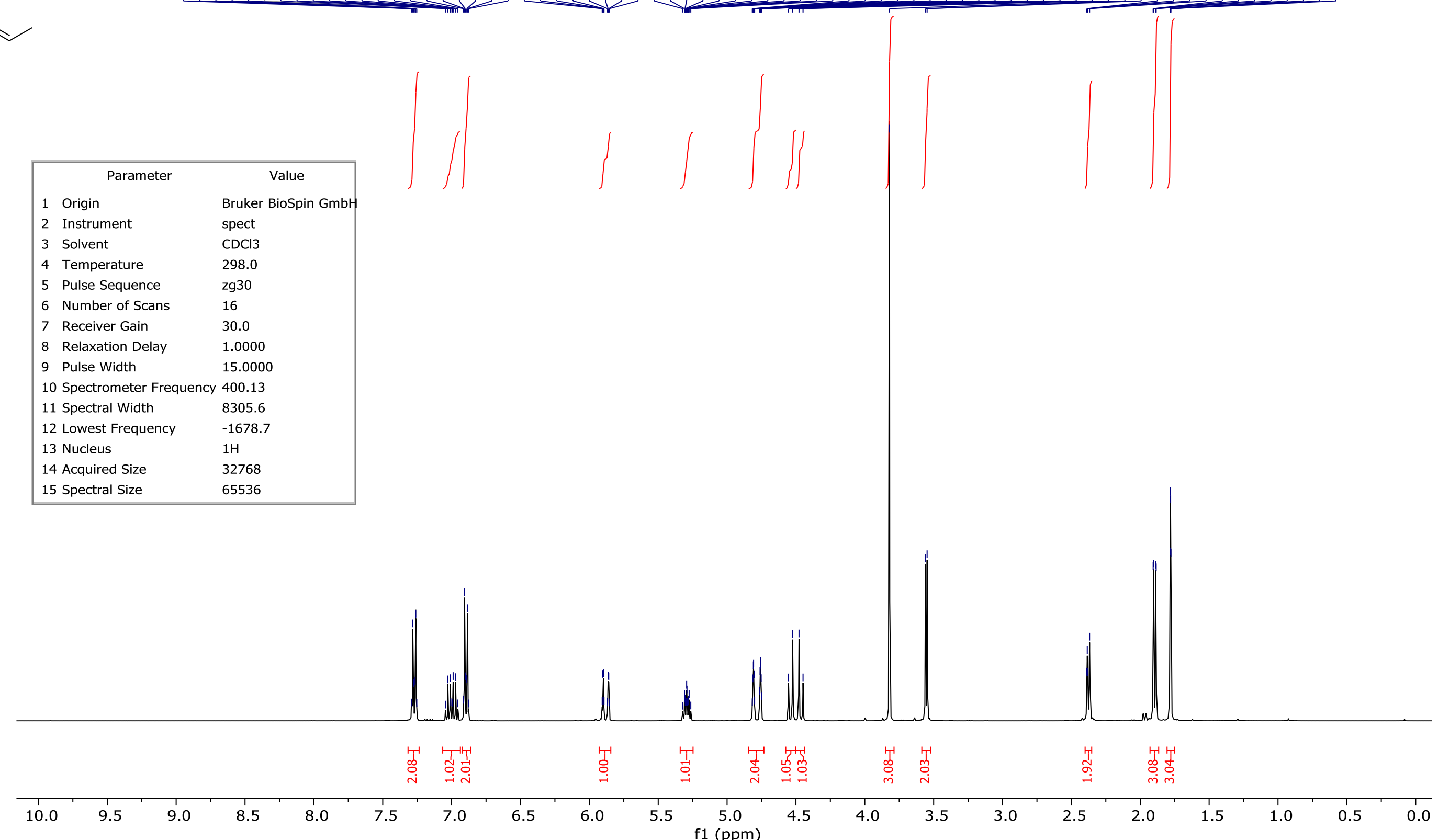


\section{${ }^{13} \mathrm{C}-\mathrm{NMR}$ compound SI-20}

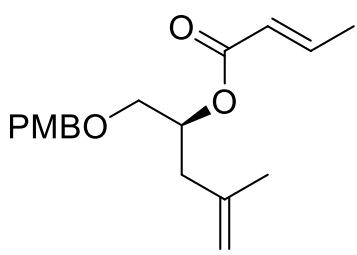

\begin{tabular}{|lll|}
\hline & \multicolumn{1}{c|}{ Parameter } & \multicolumn{1}{c|}{ Value } \\
1 & Origin & Bruker BioSpin GmbH \\
2 & Instrument & spect \\
3 & Solvent & CDCl3 \\
4 & Temperature & 298.0 \\
5 & Pulse Sequence & udeft \\
6 & Number of Scans & 128 \\
7 & Receiver Gain & 197.6 \\
8 & Relaxation Delay & 2.0000 \\
9 & Pulse Width & 9.4000 \\
10 & Spectrometer Frequency & 100.62 \\
11 & Spectral Width & 26041.7 \\
12 & Lowest Frequency & -942.5 \\
13 & Nucleus & $13 C$ \\
14 & Acquired Size & 32768 \\
15 & Spectral Size & 65536 \\
\hline
\end{tabular}

15 Spectral Size

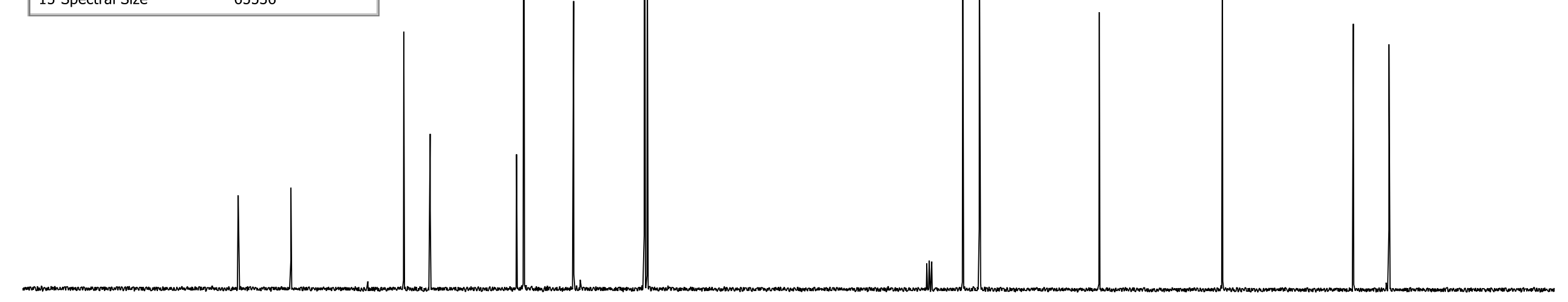




\section{${ }^{1} \mathrm{H}-\mathrm{NMR}$ compound SI-21}<smiles>C=C(C)CC(CO)OC(=O)/C=C/C</smiles>

\begin{tabular}{|lll|}
\hline \multicolumn{1}{|c|}{ Parameter } & \multicolumn{2}{c|}{ Value } \\
1 & Origin & Bruker BioSpin GmbH \\
2 & Instrument & spect \\
3 & Solvent & CDCl3 \\
4 & Temperature & 298.0 \\
5 & Pulse Sequence & zg30 \\
6 & Number of Scans & 16 \\
7 & Receiver Gain & 30.0 \\
8 & Relaxation Delay & 1.0000 \\
9 & Pulse Width & 15.0000 \\
10 & Spectrometer Frequency & 400.13 \\
11 & Spectral Width & 8305.6 \\
12 & Lowest Frequency & -1691.2 \\
13 & Nucleus & $1 \mathrm{H}$ \\
14 & Acquired Size & 32768 \\
15 & Spectral Size & 65536 \\
\hline
\end{tabular}

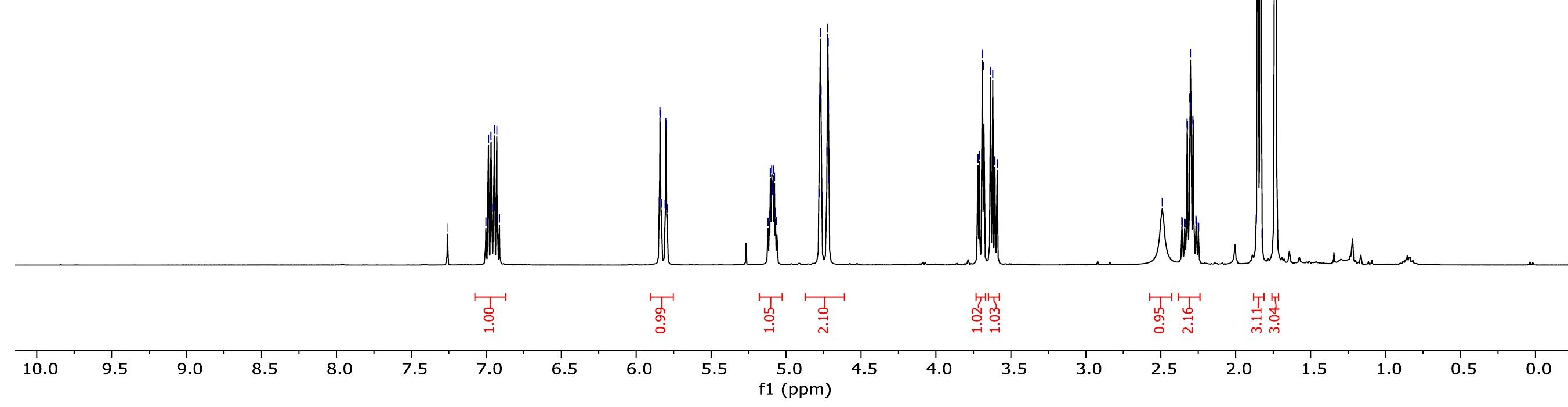




\section{${ }^{13}$ C-NMR compound SI-21}

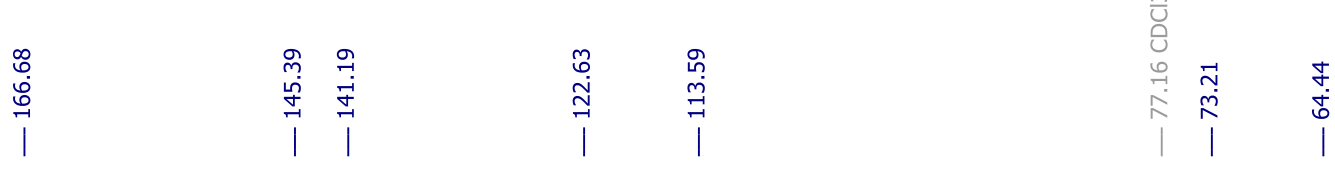<smiles>C=C(C)CC(CO)OC(=O)/C=C/C</smiles>

\begin{tabular}{|lll|}
\hline \multicolumn{1}{|c|}{ Parameter } & \multicolumn{1}{c|}{ Value } \\
1 & Origin & Bruker BioSpin GmbH \\
2 & Instrument & spect \\
3 & Solvent & CDCI3 \\
4 & Temperature & 298.0 \\
5 & Pulse Sequence & udeft \\
6 & Number of Scans & 128 \\
7 & Receiver Gain & 197.6 \\
8 & Relaxation Delay & 2.0000 \\
9 & Pulse Width & 9.4000 \\
10 & Spectrometer Frequency 100.61 \\
11 & Spectral Width & 26041.7 \\
12 & Lowest Frequency & -941.1 \\
13 & Nucleus & $13 \mathrm{C}$ \\
14 & Acquired Size & 32768 \\
15 & Spectral Size & 32768 \\
\hline
\end{tabular}




\section{${ }^{1} \mathrm{H}-\mathrm{NMR}$ compound $\mathbf{1 2}$}

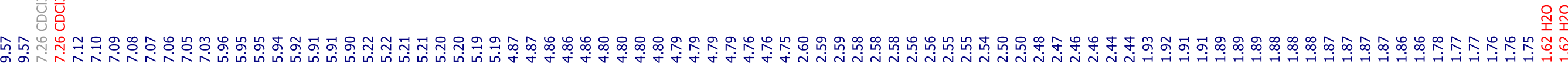

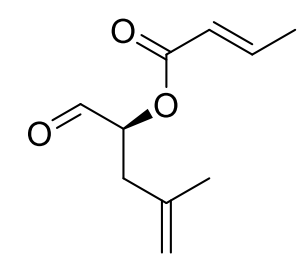

\begin{tabular}{|lll|}
\hline \multicolumn{1}{|c|}{ Parameter } & \multicolumn{1}{c|}{ Value } \\
1 & Origin & Bruker BioSpin Gmb \\
2 & Instrument & spect \\
3 & Solvent & CDCl3 \\
4 & Temperature & 298.0 \\
5 & Pulse Sequence & $\mathrm{zg} 30$ \\
6 & Number of Scans & 16 \\
7 & Receiver Gain & 111.0 \\
8 & Relaxation Delay & 1.0000 \\
9 & Pulse Width & 15.0000 \\
10 & Spectrometer Frequency & 400.13 \\
11 & Spectral Width & 8305.6 \\
12 & Lowest Frequency & -1691.6 \\
13 & Nucleus & $1 \mathrm{H}$ \\
14 & Acquired Size & 32768 \\
15 & Spectral Size & 65536 \\
\hline
\end{tabular}
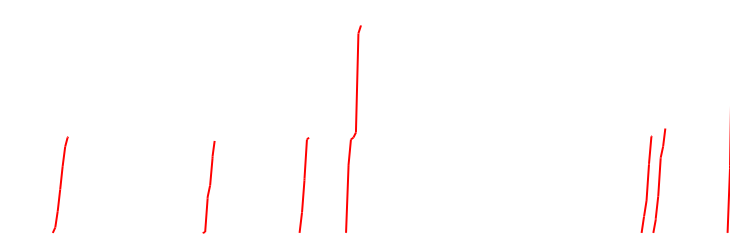

15 Spectral Size

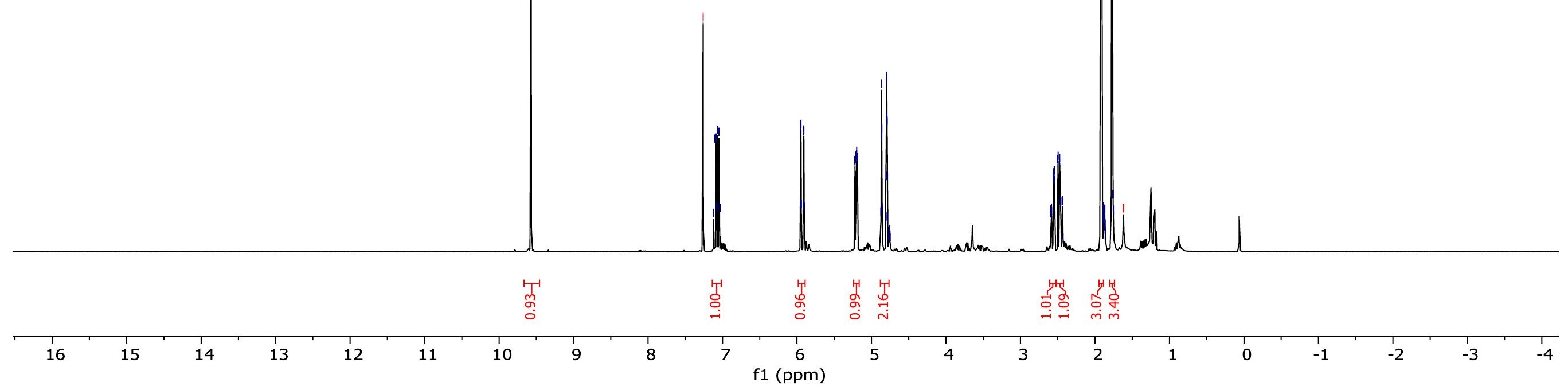




\section{${ }^{13} \mathrm{C}-\mathrm{NMR}$ compound 12}

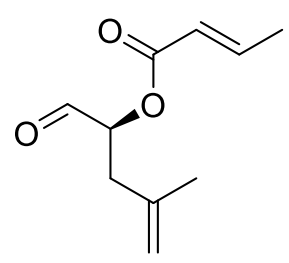

\begin{tabular}{|lll|}
\hline \multicolumn{1}{|c|}{ Parameter } & \multicolumn{1}{c|}{ Value } \\
1 & Origin & Bruker BioSpin GmbH \\
2 & Instrument & spect \\
3 & Solvent & $\mathrm{CDCl}$ \\
4 & Temperature & 298.0 \\
5 & Pulse Sequence & udeft \\
6 & Number of Scans & 128 \\
7 & Receiver Gain & 197.6 \\
8 & Relaxation Delay & 2.0000 \\
9 & Pulse Width & 9.4000 \\
10 & Spectrometer Frequency & 100.61 \\
11 & Spectral Width & 26041.7 \\
12 & Lowest Frequency & -934.2 \\
13 & Nucleus & $13 \mathrm{C}$ \\
14 & Acquired Size & 32768 \\
15 & Spectral Size & 32768 \\
\hline
\end{tabular}

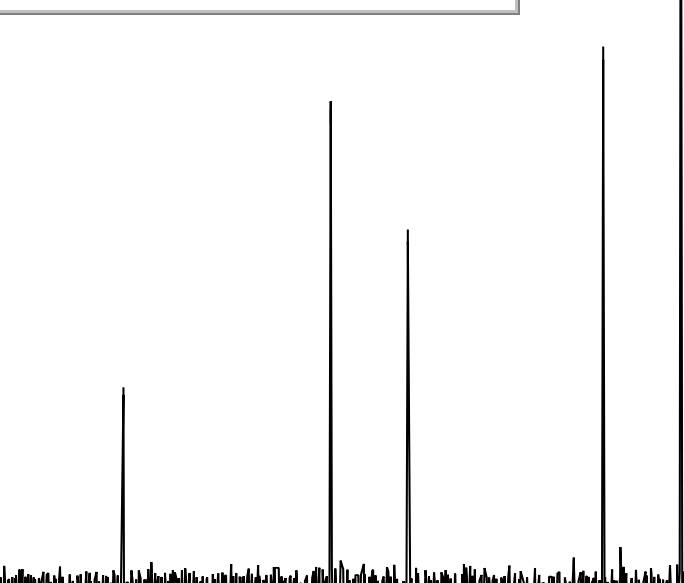




\section{${ }^{1} \mathrm{H}-\mathrm{NMR}$ compound 14}

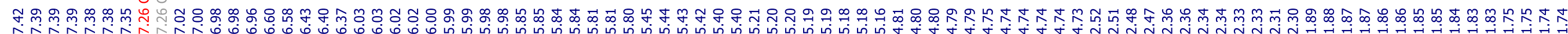

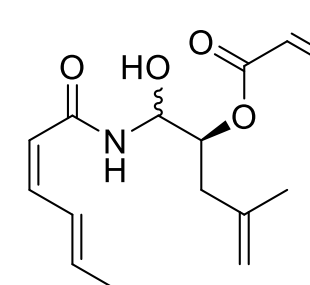

\begin{tabular}{|lll|}
\hline \multicolumn{1}{|c|}{ Parameter } & \multicolumn{2}{c|}{ Value } \\
1 & Origin & Bruker BioSpin GmbH \\
2 & Instrument & spect \\
3 & Solvent & CDCl3 \\
4 & Temperature & 298.0 \\
5 & Pulse Sequence & $\mathrm{zg} 30$ \\
6 & Number of Scans & 16 \\
7 & Receiver Gain & 70.3 \\
8 & Relaxation Delay & 1.0000 \\
9 & Pulse Width & 15.0000 \\
10 & Spectrometer Frequency & 400.13 \\
11 & Spectral Width & 8305.6 \\
12 & Lowest Frequency & -1691.5 \\
13 & Nucleus & $1 \mathrm{H}$ \\
14 & Acquired Size & 32768 \\
15 & Spectral Size & 65536 \\
\hline
\end{tabular}
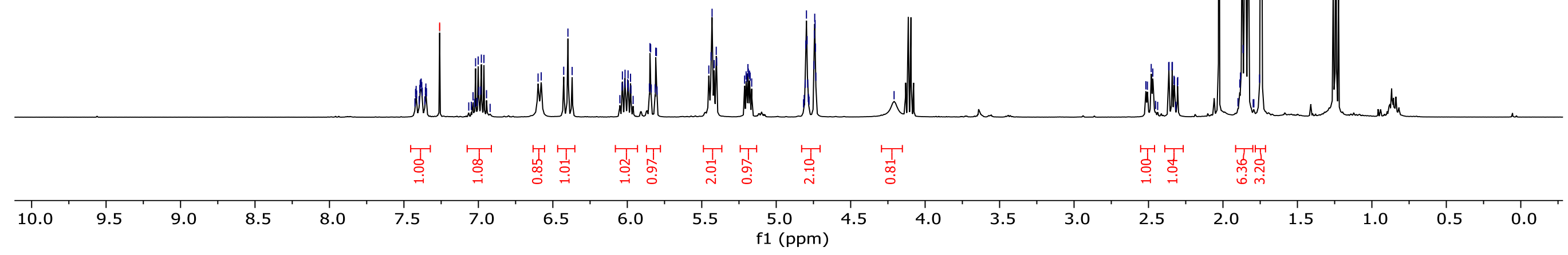


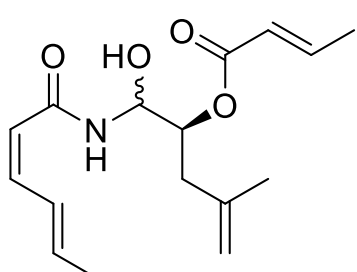

\begin{tabular}{|lll|}
\hline \multicolumn{1}{|c|}{ Parameter } & \multicolumn{1}{c|}{ Value } \\
1 & Origin & Bruker BioSpin GmbH \\
2 & Instrument & spect \\
3 & Solvent & $\mathrm{CDCl}$ \\
4 & Temperature & 298.0 \\
5 & Pulse Sequence & udeft \\
6 & Number of Scans & 128 \\
7 & Receiver Gain & 197.6 \\
8 & Relaxation Delay & 2.0000 \\
9 & Pulse Width & 9.4000 \\
10 & Spectrometer Frequency & 100.61 \\
11 & Spectral Width & 26041.7 \\
12 Lowest Frequency & -935.8 \\
13 Nucleus & $13 \mathrm{C}$ \\
14 Acquired Size & 32768 \\
15 & Spectral Size & 32768 \\
\hline
\end{tabular}

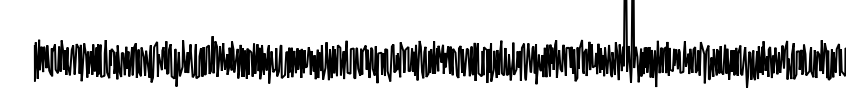

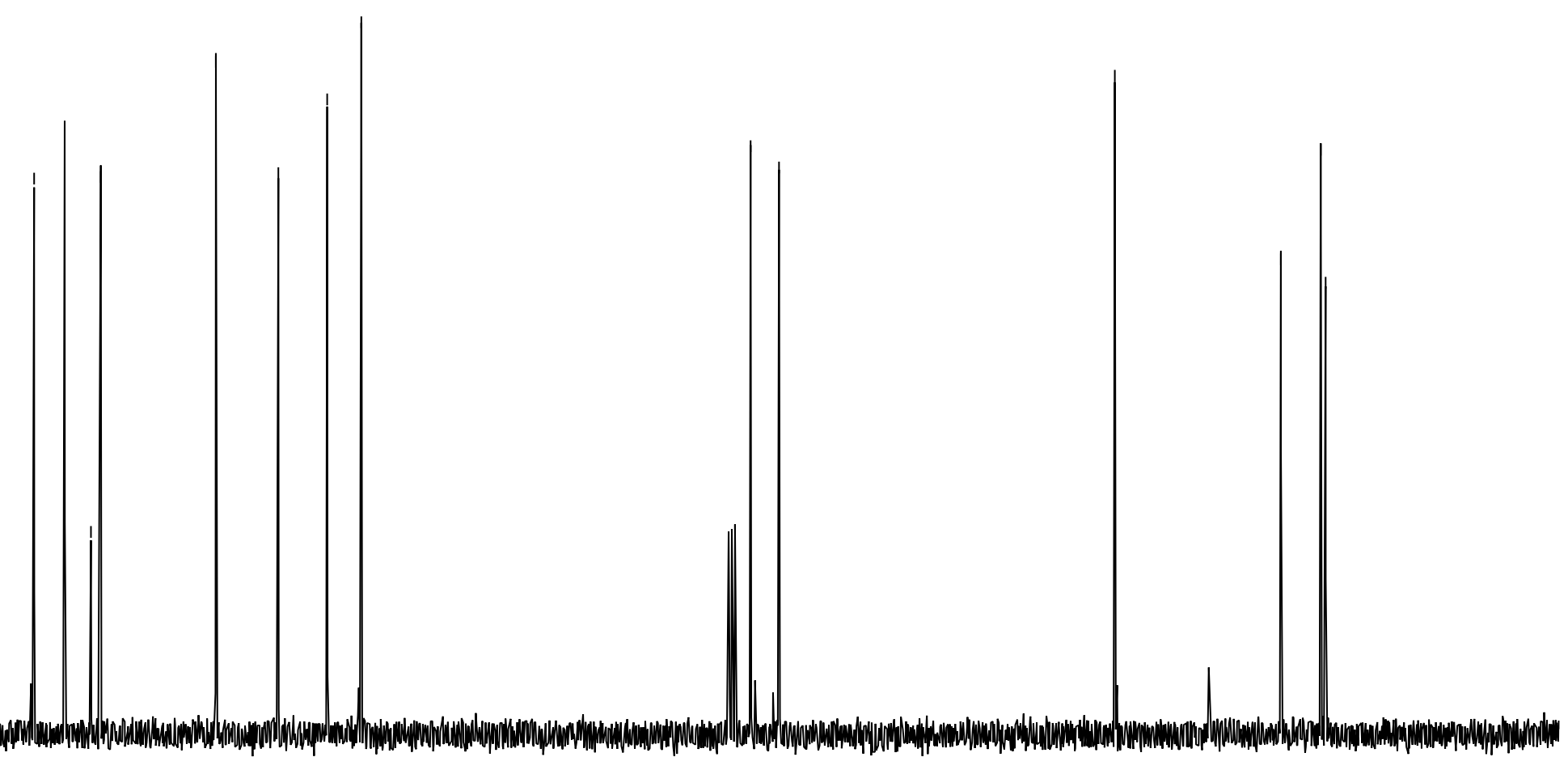

